\title{
Identification of transcriptional regulatory elements for Ntng1 and Ntng2 genes in mice
}

\author{
Kunio Yaguchi ${ }^{1,2}$, Sachiko Nishimura-Akiyoshi ${ }^{1}$, Satoshi Kuroki ${ }^{1}$, Takashi Onodera ${ }^{2}$ and Shigeyoshi Itohara ${ }^{1 *}$
}

\begin{abstract}
Background: Higher brain function is supported by the precise temporal and spatial regulation of thousands of genes. The mechanisms that underlie transcriptional regulation in the brain, however, remain unclear. The Ntng 1 and Ntng2 genes, encoding axonal membrane adhesion proteins netrin-G1 and netrin-G2, respectively, are paralogs that have evolved in vertebrates and are expressed in distinct neuronal subsets in a complementary manner. The characteristic expression patterns of these genes provide a part of the foundation of the cortical layer structure in mammals,

Results: We used gene-targeting techniques, bacterial artificial chromosome (BAC)-aided transgenesis techniques, and in vivo enhancer assays to examine transcriptional mechanisms in vivo to gain insight into how the characteristic expression patterns of these genes are acquired. Analysis of the gene expression patterns in the presence or absence of netrin-G1 and netrin-G2 functional proteins allowed us to exclude the possibility that a feedback or feedforward mechanism mediates their characteristic expression patterns. Findings from the BAC deletion series revealed that widely distributed combinations of cis-regulatory elements determine the differential gene expression patterns of these genes and that major cis-regulatory elements are located in the 85-45 kb upstream region of Ntng2 and in the 75-60 kb upstream region and intronic region of Ntng1. In vivo enhancer assays using 2-kb evolutionarily conserved regions detected enhancer activity in the distal upstream regions of both genes.

Conclusions: The complementary expression patterns of Ntng 1 and Ntng2 are determined by transcriptional cis-regulatory elements widely scattered in these loci. The cis-regulatory elements characterized in this study will facilitate the development of novel genetic tools for functionally dissecting neural circuits to better understand vertebrate brain function.
\end{abstract}

\section{Background}

Development and function of neural circuits in the vertebrate brain are supported by the orchestration of the spatial and temporal expression of genes in the brain. Disturbances in the transcriptional mechanisms are associated with major neurologic disorders, including mental deficiency, cerebral palsy, epilepsy, schizophrenia, and autism [1,2]. The characterization of the cis-acting regulatory sequences supporting proper gene expression, however, is insufficient. Unlike coding-sequences, distant cis-acting transcription regulatory sequences involved in particular biologic processes are difficult to identify because they locate in the vast and poorly characterized non-coding portion of the genome and may be located

\footnotetext{
* Correspondence: sitohara@brain.riken.jp

${ }^{1}$ RIKEN Brain Science Institute, 2-1 Hirosawa, Wako, Saitama, Japan Full list of author information is available at the end of the article
}

hundreds or thousands of kilobase (kb) pairs away from the target genes they regulate [3]. Therefore, the identification of sequences that control the location and timing of gene expression in the brain is a crucial challenge in neuroscience.

Netrin-G1 (Ntng1) and netrin-G2 (Ntng2), also called laminet 1 and laminet 2, respectively, are UNC-6/netrin family members [4-6]. Classic netrins are phylogenetically conserved diffusible chemoattractants of axon guidance molecules [7-9]. Unlike classic netrins, netrin-Gs are linked to the plasma membrane surface by a glycosylphosphatidylinositol linkage, have no invertebrate orthologs, and lack affinity to the known netrin receptor families $[4,5]$. Netrin-G1 and netrin-G2 interact with specific receptors. Netrin-G1 interacts with netrin-G1 ligand (NGL1) [10], whereas netrin-G2 interacts with NGL2 [11,12]. Ntng1 and Ntng2 are clearly expressed in 
distinct neuronal subsets in a complementary manner [5,6] and have different roles in distinct neuronal circuits $[12,13]$. The differential expression patterns are highly conserved in primates such as marmosets and macaque [14], and also likely in humans [15]. Human genetics studies have detected single nucleotide polymorphisms in both NTNG1 and NTNG2 in association with schizophrenia [16-18] and rearrangements in NTNG1 in a patient with Rett syndrome [19,20]. NTNG1 and NTNG2 alterations might also be involved in bipolar disease [21,22]. Studies with netrin-G1 and netrin-G2 knock-out (KO) mice have revealed the crucial significance of their differential expression in higher brain functions [12]. Elucidation of the transcriptional mechanisms that regulate the complementary expression of Ntng1 and Ntng2 will help to clarify the mechanisms of vertebrate-specific neural circuit formation and function, and will act as a springboard for novel cutting-edge research designed to gain a better understanding of the basis of higher brain function in vertebrates.

Mouse Ntng1 and Ntng2 are large genes that span more than $362 \mathrm{~kb}$ and $53 \mathrm{~kb}$, respectively. Their cis-regulatory elements may also be widely distributed. The insert size limitation of high-copy plasmid constructs makes the use of these plasmids impractical for identifying more distant cis-regulatory elements. Bacterial artificial chromosomes (BACs), however, allow for the modification of genomic DNA, including large areas $(\sim 200 \mathrm{~kb})$ that can cover the whole gene locus [23,24]. BAC DNA recombination methods have also been improved [25]. Moreover, recent progress in genome research allows for the identification of Evolutionarily Conserved Regions (ECRs) among species throughout their genomes. ECRs are useful for predicting functional domains without prior knowledge of the actual function [26-28]. Taking advantage of these tools, we constructed BAC transgenic mice to analyze the molecular mechanisms that underlie the regulation of Ntng1 and Ntng2 transcription.

In the present study, we analyzed the transcriptional mechanisms that regulate the characteristic expression patterns of Ntng1 and Ntng2. First, we examined the transcriptional properties of Ntng1 and Ntng2 based on the expression of the Escherichia coli beta-galactosidase gene (LacZ) in knock-in (KI) mice. Second, to examine possible trans-mechanisms for the differential expression in Ntng1 and Ntng2 genes, we investigated LacZ reporter expression in the presence and absence of netrin-G1 or netrin-G2. Third, to identify the cis-regulatory elements of Ntng1 and Ntng2, we performed expression analyses of both genes using a BAC transgenic mouse technique and transgenic enhancer assays with a heterologous minimal promoter in mice. Our findings revealed that cis-elements that are widely and distantly distributed from the transcription start site (TSS) determine the unique expression patterns of these genes.

\section{Results}

LacZ-KI mice exhibit complementary expression patterns of Ntng 1 and Ntng2

To investigate the spatial distribution of Ntng1 and Ntng2 expression in detail, we generated Ntng1- and Ntng2$L a c Z$-KI mice. These mice carry $L a c Z$ fused with a nuclear localization signal and an SV40 polyadenylation signal (NLS-LacZ-pA) in-frame in exon 2 of the Ntng1 or Ntng2 gene, and thus the transcriptional activities of these genes can be monitored by examining $\operatorname{Lac} Z$ activity at a single cell resolution. We performed 5-bromo-4-chloro-3-indolyl$\beta$-d-galactoside (X-gal) staining of Ntng1- and Ntng2-LacZKI mouse brains at postnatal day 21 (P21). The expression patterns of these genes are largely maintained throughout life. The findings from the Ntng1-LacZ-KI mice and Ntng2LacZ-KI mice are summarized in Additional file 1: Table S1, and representative photos are shown in Figure 1 and Additional file 2: Figure S1.

In the cerebral cortex, Ntng1 was expressed in neurons in layer V (Figure 1C to G, Additional file 2: Figure S1C), whereas Ntng2 was expressed in neurons in layers II/III, IV, and VI (Figure 1N to S, Additional file 2: Figure S1H). The claustrum and endopiriform nucleus are closely associated with the cortex. Consistent with data from a previous study in monkeys and rats [14], only Ntng2 was expressed in the claustrum and endopiriform nucleus (Figure $1 \mathrm{O}$ to Q). In the olfactory areas, Ntng1 was highly expressed in the olfactory bulb mitral cells and glomerular cells (Figure 1A). Only Ntng2 was expressed in the taenia tecta (Figure 1N). In the anterior olfactory nucleus, Ntng1 was expressed in the neurons of dorsal, lateral, and medial parts (Figure 1B, Additional file 2: Figure S1A), whereas Ntng2 was expressed in the neurons of the external part (Figure 1M, Additional file 2: Figure S1F). In the piriform cortex, Ntng1 was expressed in layer II neurons (Figure 1C to G, Additional file 2: Figure S1B), and Ntng2 was expressed in layer III neurons (Figure $1 \mathrm{~N}$ to $\mathrm{R}$, Additional file 2: Figure S1G). Ntng2 was expressed in dentate granule cells and hippocampal pyramidal neurons (Figure 1Q and R). Ntng1, but not Ntng2, was strongly expressed in the subiculum (Figure $1 \mathrm{H}$ and S). Conversely, Ntng2, but not Ntng1, was strongly expressed in the parasubiculum, postsubiculum, and presubiculum (Figure $1 \mathrm{H}$ and S). In the entorhinal area, Ntng1 was expressed in layer II of the lateral entorhinal area and layer III throughout the entorhinal area (Additional file 2: Figure S1D), and Ntng2 was expressed at a relatively low level in layer II of the medial entorhinal area and layer III of the lateral entorhinal area (Additional file 2: Figure S1I). In the amygdala, Ntng1 was expressed in the lateral amygdala nucleus (Figure 1F, Additional file 2: Figure S1E), whereas Ntng2 was expressed in the basolateral amygdala nucleus (Figure 1Q, Additional file 2: Figure S1J). In the striatum, Ntng1 and Ntng2 were expressed in the olfactory tubercles 


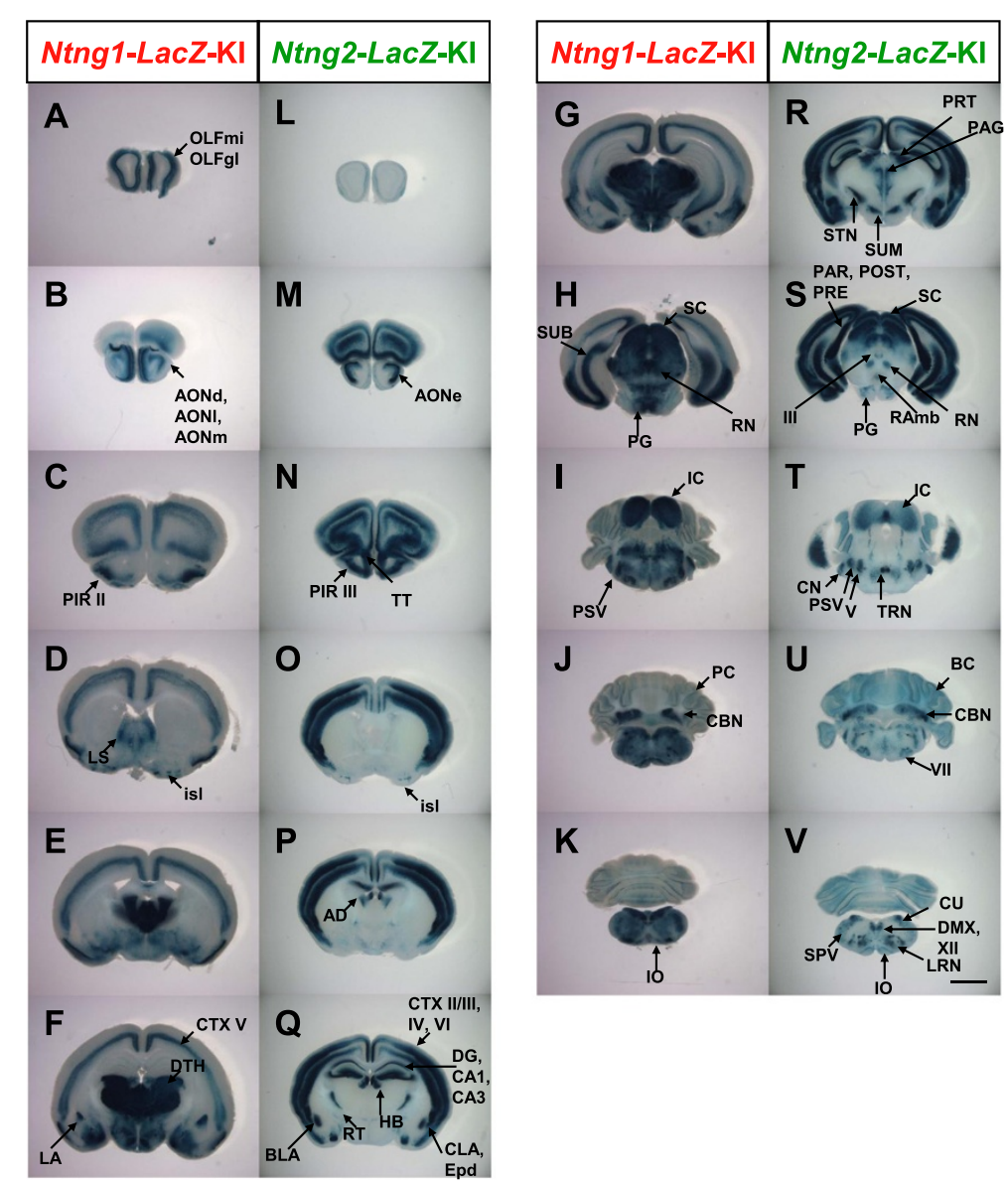

Figure 1 X-gal staining of Ntng1- and Ntng2-LacZ-KI mouse brains. (A-K) X-gal staining of Ntng 1-LacZ-KI mouse brains. (L-V) X-gal staining of Ntng2-LacZ-KI mouse brains. Coronal slices $(400 \mu \mathrm{m})$ at P21 were stained with X-gal staining solution. In panels A-K and $L-V$, the stained sections are arranged from anterior to posterior. $A D$, anterodorsal nucleus of the thalamus; $A O N$, anterior olfactory nucleus; $B C$, Bergmann glia cells; BLA, basolateral amygdala nucleus; CA1, cornu ammonis 1 field of the hippocampus; CA3, cornu ammonis 3 field of the hippocampus; CBN, cerebellar nuclei; CLA, claustrum; CN, cochlear nuclei; CTX, cerebral cortex; CU, cuneate nucleus; DG, dentate gyrus; DMX, dorsal motor nucleus of the vagus nerve; DTH, dorsal thalamus; ENT, entorhinal area; Epd, endopiriform nucleus, dorsal part; HB, habenula; IC, inferior colliculus; isl, olfactory tubercle; Islands of Calleja; IO, inferior olivary complex; LA, lateral amygdala nucleus; LRN, lateral reticular nucleus; LS, lateral septal nucleus; OLFgl, olfactory bulb, glomerular layer; OLFmi, olfactory bulb, mitral layer; PAG, periaqueductal gray, dorsal division; PC, Purkinje cell; PIR, piriform area; $\mathrm{PG}$, pontine gray; PRT, pretectal region; PSV, principal sensory nucleus of the trigeminal; RAmb, midbrain raphe nuclei; RN, red nucleus; RT, reticular nucleus of the thalamus; SC, superior colliculus; SPV, spinal nucleus of the trigeminal; STN, subthalamic nucleus; SUB, subiculum; SUM, supramammillary nucleus; TRN, tegmental reticular nucleus; $T$, taenia tecta; II, oculomotor nucleus; $V$, trigeminal motor nucleus; VII, facial motor nucleus; XII, hypoglossal nucleus. Scale bar: $2.0 \mathrm{~mm}$ for all panels.

and Islands of Calleja (Figure 1D and O). Ntng1 was modestly expressed in the lateral and medial septal nuclei (Figure 1D). In the cerebellum, Ntng1 and Ntng2 were strongly expressed in the cerebellar nuclei (Figure $1 \mathrm{~J}$ and $\mathrm{U}$ ). Ntng2 was modestly expressed in the Bergmann glia (Figure 1U). Ntng1 was weakly expressed in the Purkinje cells (Figure 1J). In the thalamus and peri-thalamic region, Ntng1 was expressed in most nuclei except for the reticular thalamic nucleus and the habenula (Figure 1F), whereas complementary Ntng2 expression was observed in the reticular thalamic nucleus, medial and lateral habenula, and anterodorsal nucleus of the thalamus (Figure 1P and Q). In the hypothalamus, Ntng1 was expressed in the medial mammillary nucleus, whereas Ntng2 was highly expressed in the supramammillary nucleus and subthalamic nucleus (Figure 1R). In the midbrain, although both genes were expressed in the superior and inferior colliculi (Figure $1 \mathrm{H}$, $\mathrm{I}, \mathrm{S}$, and $\mathrm{T}$ ), the expression gradients differed in distinct layers of these structures. The red nucleus expressed both genes (Figure $1 \mathrm{H}$ and S). Ntng2 expression was detected in the dorsal division of the periaqueductal gray, pretectal region, oculomotor nucleus, and midbrain raphe nuclei (Figure $1 \mathrm{R}$ and $\mathrm{S}$ ). In the pons, both genes were expressed in the pontine gray and principal sensory nucleus of the trigeminal nerve (Figure 1H, I, S, and T). Ntng2 was further expressed in the tegmental reticular nucleus, trigeminal 
motor nucleus, and facial motor nucleus (Figure 1T). In the medulla, both genes were expressed in the inferior olivary complex (Figure $1 \mathrm{~K}$ and V). Ntng2 was modestly expressed in the cochlear nuclei, cuneate nucleus, spinal trigeminal nucleus, dorsal motor nucleus of the vagus nerve, lateral reticular nucleus, and hypoglossal nucleus (Figure $1 \mathrm{U}$ and V).

Consistent with previous in situ hybridization findings [4-6], X-gal staining for Ntng1- and Ntng2-LacZ-KI mice revealed differential expression patterns of Ntng1 and Ntng2 in most brain regions.

\section{Examination of gene regulatory mechanisms between Ntng1 and Ntng2 acting in trans}

We hypothesized that the complementary expression of Ntng1 and Ntng2 is regulated by trans-acting mechanisms in a manner similar to the expression of olfactory receptor (OR) [29], immunoglobulin, and T-cell receptor genes [30,31]. In allelic exclusion, in the immune system, a functional $\mathrm{V}(\mathrm{D}) \mathrm{J}$ rearrangement in one allele suppresses rearrangements in another allele by negative feedback regulation. One particular OR gene is activated by a cis-acting locus control region ( $\mathrm{H}$ region), and functional OR molecules transmit inhibitory signals to block further activation of additional OR genes. For example, functional expression of netrin-G1 protein might inhibit Ntng2 transcription, and that of netrin-G2 protein might inhibit Ntng1 transcription. Alternatively, they may also accelerate the expression of their own genes. We tested this possibility by examining expression patterns in the $L a c Z$ KI allele in the absence of netrin-G1 or netrin-G2. We interbred Ntng-LacZ-KI mice and other Ntng1-KO or Ntng2-KO variants and investigated the expression patterns of the $L a c Z$ gene in the presence or absence of netrin-G1 or netrin-G2. We observed no changes in LacZ expression in the Ntng1- and Ntng2-LacZKI alleles under these conditions (Figure 2). These results clearly ruled out the possibility that trans mechanisms underlie the differential expression of Ntng1 and Ntng2.

\section{Cis-regulatory elements responsible for endogenous Ntng2 expression}

To systematically examine the transcriptional mechanisms underlying the characteristic expression patterns of Ntng2 in young adult mouse brains, the cis-regulatory elements of Ntng2 were analyzed in BAC transgenic mice. The Ntng2BAC clone (RP23-417D10) that covered the 195-kb region of the locus between $-95 \mathrm{~kb}$ and $+100 \mathrm{~kb}$ of the Ntng2 TSS was used to generate Ntng2-LacZ-BAC transgenic mice (Figure 3). The adjacent genes, Med27 and Setx, locate at $94 \mathrm{~kb}$ upstream and $70 \mathrm{~kb}$ downstream of the Ntng2 TSS, respectively.

Using the Red/ET recombination technique [32], the Ntng2-BAC clone was inserted with an NLS-LacZ-pA cassette at the translation start site in exon 2 . The resulting Ntng2-LacZ-BAC construct was microinjected into the pronuclei of fertilized mouse eggs to establish transgenic founders. Transgenic founder mice were transcardially perfused with $4 \%$ paraformaldehyde at P21. Fixed mouse brains were sliced at a thickness of $400 \mu \mathrm{m}$ and stained for $L a c Z$ activity using X-gal. Only founders that carried the end sequences of the transgene were selected and analyzed. Throughout this study, the same protocol for detecting $L a c Z$ expression was used to analyze expression patterns in transgenic mice.

In the Ntng2-LacZ-BAC transgenic mice, LacZ expression was reproducibly detected in the cerebral cortex layers II/III, IV, and VI, anterior olfactory nucleus, piriform area layers III, hippocampal areas CA1 and CA3, dentate gyrus, basolateral amygdala nucleus, Bergmann glia cells, cerebellar nuclei, anterodorsal nucleus of the thalamus, reticular nucleus of the thalamus, habenula, superior colliculus, and inferior colliculus (Figure 4A; Additional file 3: Table S2). These expression patterns of $L a c Z$ in the Ntng2-LacZ-BAC transgenic mice were similar to those in the Ntng2-LacZ-KI mice (Figure 1L-V), indicating that the Ntng2-LacZBAC construct contained physiologically significant cisregulatory elements for endogenous Ntng2 expression.

To determine the critical regions for regulatory activity, the 195-kb region of the Ntng2 locus was divided into six segments (I-VI) by considering the ECRs among species-mouse and human (Figure 3 and Additional file 4: Figure S2). This homology alignment was performed using a VISTA homology search program [33]. The adjacent gene, Med27, was also taken into consideration. Ntng2 segments I-VI represent the -95 to $-85 \mathrm{~kb},-85$ to $-45 \mathrm{~kb},-45$ to $-23 \mathrm{~kb},-23$ to $-6 \mathrm{~kb}$, and the end of Ntng 2 exon2 to $+100 \mathrm{~kb}$ positions of the Ntng 2 TSS, respectively. The corresponding series of deletion constructs were referred to as Ntng2-Del I, II, III, IV, I-IV, VI, and I-IV\&VI, respectively. Representative results from the Ntng2 deletion series are shown in Figure 4. If the frequency of LacZ expression in a given brain area exceeded $50 \%$ of the sample, we considered that the construct carried a cis-element for a given brain area (Additional file 3: Table S2).

The deletion of segment I did not affect $L a c Z$ expression in any brain region examined (Figure 4B, Additional file 3 : Table S2). Deletion of segment II led to a loss of LacZ expression in various brain areas, including the cerebral cortex, anterior olfactory nucleus, piriform area layers III, basolateral amygdala nucleus, reticular nucleus of the thalamus, and inferior colliculus (Figure 4C, Additional file 3: Table S2). Deletion of segment III led to a loss of LacZ expression in the reticular nucleus of the thalamus and inferior colliculus (Figure 4D, Additional file 3: Table S2). The deletion of segment IV did not affect $L a c Z$ expression in any brain area examined (Figure 4E, Additional file 3: Table S2). Large deletion of segments I-IV led to a loss of $\mathrm{LacZ}$ expression in CA1, CA3, and the superior colliculus, as well as brain areas affected by the deletion of a single 

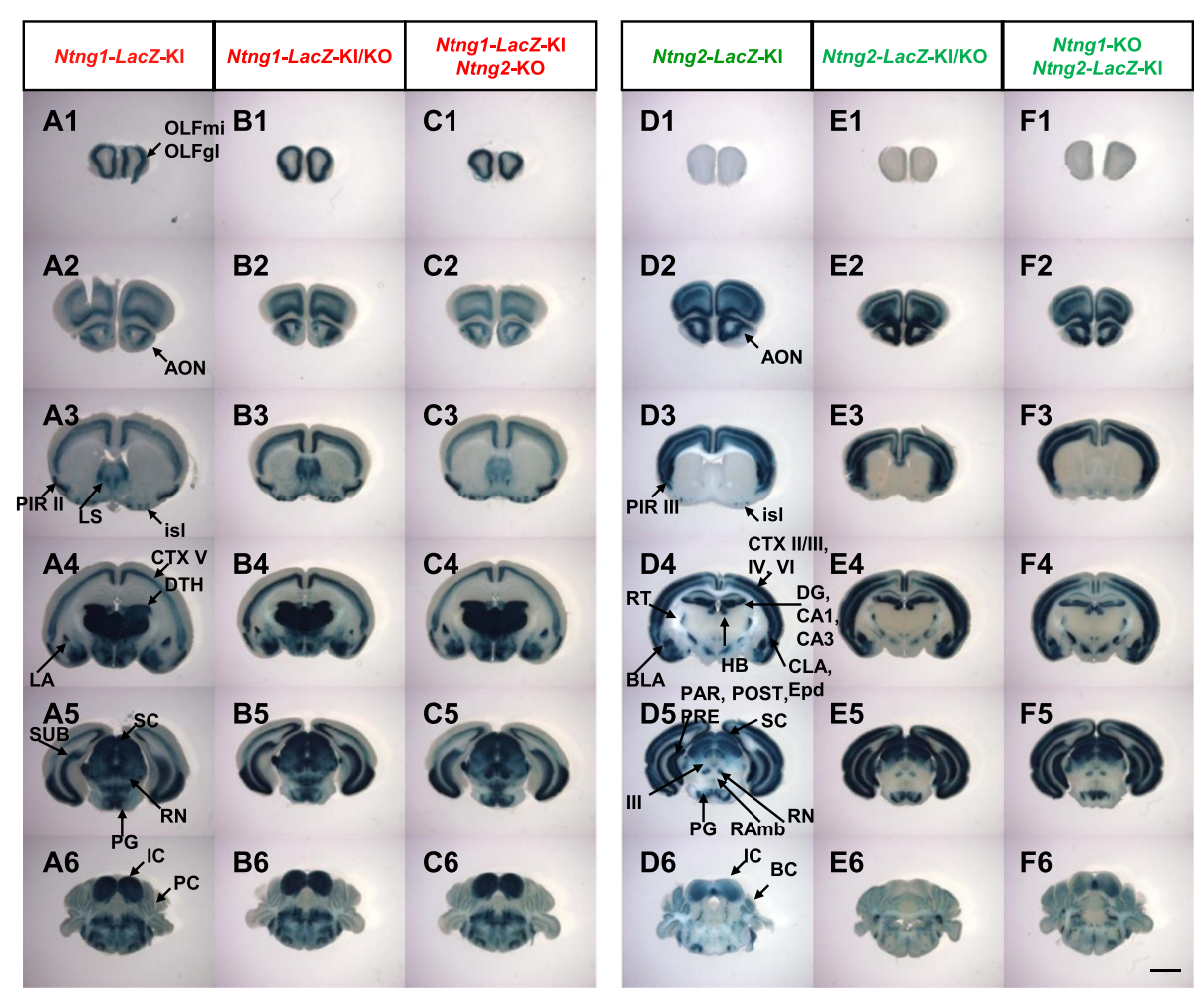

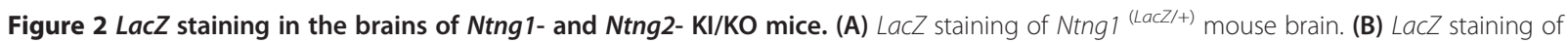
Ntng $1^{\text {(LacZ/GFP) }}$ mouse brain. This mouse lacked netrin-G1. (C) LacZ staining of Ntng $1^{(\text {LacZ/+) }}$ and Ntng $2^{(-/-)}$mouse brain. (D) LacZ staining of

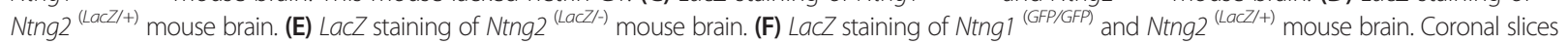
$(400 \mu \mathrm{m})$ at P21 were stained with X-gal solution. The panels are arranged from anterior (Top) to posterior (Bottom). Scale bar: $2.0 \mathrm{~mm}$ for all panels.

segment II or III (Figure 4 F, Additional file 3: Table S2). The deletion of segment VI only affected LacZ expression in the reticular nucleus of the thalamus and habenula (Figure 4G, Additional file 3: Table S2). The combined deletion of segments I-IV and VI led to a complete loss of the transcriptional activity of the Ntng2 promoter (Figure 4H, Additional file 3: Table S2). These data from the Ntng2 BAC deletion series suggest that multiple enhancers are widely distributed upstream and downstream of the TSS site and that a major cis-element is located in segment II (from -85 to $-45 \mathrm{~kb}$ of Ntng2) (Figure 5A). These findings also suggest that multiple cis-elements cooperate to regulate the transcriptional activities in several brain areas, such as hippocampus, anterodorsal thalamus, superior colliculus, cerebellar nuclei and Bergman glia (Additional file 3: Table S2).

\section{Cis-regulatory elements responsible for endogenous Ntng1 expression}

Similar studies were performed for Ntng1. We selected a BAC clone (RP23-143P6) covering the 218-kb region of the locus between $-174.9 \mathrm{~kb}$ and $+42.5 \mathrm{~kb}$ of the Ntng1 TSS (Figure 6), as a clone covering the longest region of putative transcriptional regulatory elements. The adjacent gene, Prmt6, locates 106-kb upstream of the Ntng1 TSS. The Ntng1- BAC clone was inserted with an NLS-LacZ-pA cassette at the translation start site in exon 2 . The procedures for generating and analyzing the transgenic mice were identical to those used for the Ntng2 experiment. In transgenic mice carrying the Ntng1-LacZ-BAC construct, the $L a c Z$ expression patterns resembled those of the Ntng1-KI mice, although the expression levels differed significantly in the lateral septal nucleus, cerebellar nuclei, and dorsal thalamus (Figure 7A, Additional file 5: Table S3). Subsequent studies with a series of deletion constructs, however, suggested that the lack of expression in the thalamus did not indicate the lack of a cis-element in the thalamus.

To determine the critical regions for the regulation of Ntng1 expression, we divided the 218-kb region of the Ntng1 gene locus into six segments (I-VI) by considering the ECRs and adjacent gene, Prmt6 (Figure 6A and Additional file 6: Figure S3). Ntng1 segments I-VI represent the -176 to $-75 \mathrm{~kb},-75$ to $-60 \mathrm{~kb},-60$ to $-46 \mathrm{~kb},-46$ to $-4 \mathrm{~kb},-4 \mathrm{~kb}$ to ATG of Ntng1, and at the end of Ntng1 exon 2 to $+42-\mathrm{kb}$ positions of the Ntng1 TSS, respectively. The corresponding deletion constructs were named Ntng1-Del I, II, III, IV, II-IV, VI, and I-IV\&VI, 
A

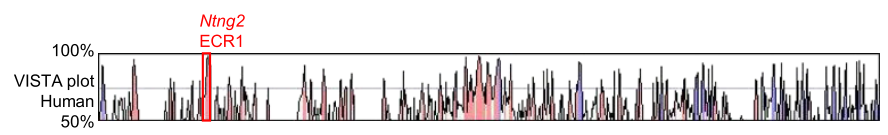

B

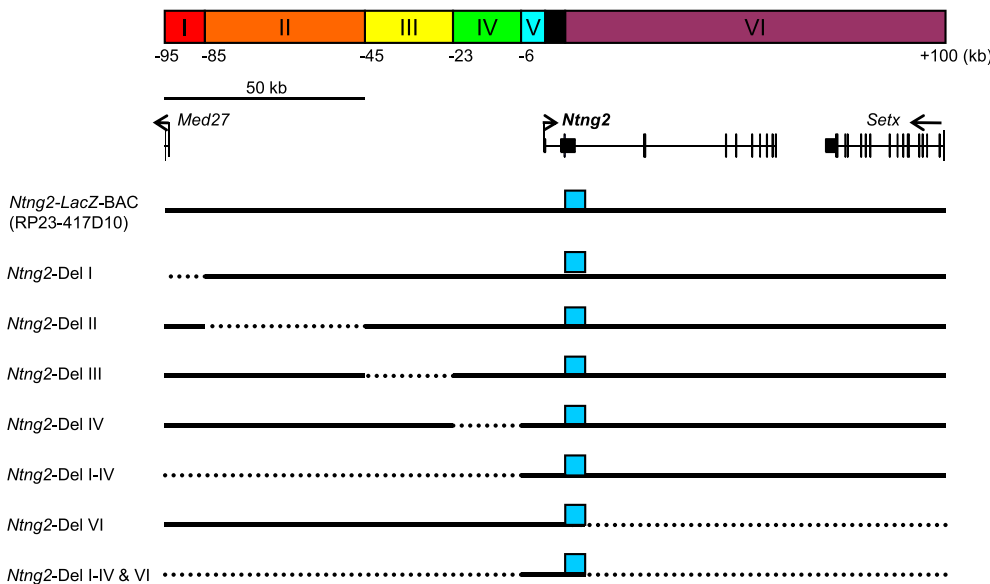

Figure 3 Highly conserved sequences in Ntng2 loci and strategy for analyzing cis-regulatory elements in transgenic mice. (A) Comparative genomic analysis of the mouse Ntng2-BAC sequence (mouse Dec. 2011 [GRCm38/mm10] assembly; chr2: 29,153,280-29,347,847) by the VISTA genome browser [http://genome.lbl.gov/vista/index.shtml] [32]. Percent nucleotide identities between mouse and human DNA sequences were plotted as a function of the position along the mouse sequence. Peaks of evolutionary conserved exons of Ntng 2 and neighboring genes are shaded blue. Aligned regions with more than $70 \%$ identity over 100 bases are shaded pink. (B) Schematic diagrams of the Ntng2 deletion series. The Ntng2-BAC sequences are divided into 6 segments (I-VI indicated in colored boxes) along with ECR distribution. Genomic organizations of the mouse Ntng2 and neighbor gene loci are indicated. Short vertical lines indicate positions of exons and arrowheads indicate transcriptional directions. Broken and solid lines represent deleted and preserved regions in the BAC constructs, respectively. Blue boxes on the lines represent the reporter LacZ cassette inserted in each BAC construct. Note that the reporter LacZ cassette, ECRs, and minimal promoter sequences are not represented in accurate scales.

respectively (Figure 6B). The results from the Ntng1 deletion series are summarized in Additional file 5: Table S3 and are shown in Figure 7.

The deletion of segment I was not associated with a loss of LacZ expression in any brain area, but additional expression was observed in the lateral septal nucleus, cerebellar nuclei, and dorsal thalamus, resembling the expression patterns of endogenous Ntng1, and suggesting negative regulatory activity in this segment (Figure 7B, Additional file 5: Table S3). Deletion of segment II led to a marked loss of expression in most brain areas, including the deep cortical layer, anterior olfactory nucleus, lateral septum, Purkinje cells, cerebellar nuclei, dorsal thalamus, and superior and inferior colliculi (Figure 7C, Additional file 5: Table S3). Deletion of segment III led to a loss of expression in the lateral septal nucleus, dorsal thalamus, and inferior colliculus (Figure 7D, Additional file 5: Table S3). Deletion of segment IV did not show substantial effects (Figure 7E, Additional file 5: Table S3). Combined deletions of segments II-IV, however, led to an almost complete loss of transcriptional activity in all brain areas (Figure 7F, Additional file 5: Table S3). Interestingly, single deletion of segment VI also led to a loss of transcriptional activity in most brain areas, except for the olfactory bulb and piriform cortex (Figure 7G,
Additional file 5: Table S3). Segment V alone did not show detectable activity (Figure 7H, Additional file 5: Table S3). These data from the Ntng1 BAC deletion series suggest that multiple enhancers are widely distributed upstream and downstream of the TSS site and that significant cis-elements locate in segment II (from -75 to $-60 \mathrm{~kb}$ of Ntng1) and segment VI (Figure 5B). These findings data also suggest that a negative regulator(s) for the lateral septal nucleus, cerebellar nuclei, and dorsal thalamus is located in segment I (Additional file 5: Table S3).

Unlike the Ntng2-BAC constructs, the Ntng1-BAC constructs showed greater variability in their reporter gene expression patterns. The variability may partly depend on the intergenic recombination of BAC constructs. For example, in the Ntng1 BAC deletion series, 5' and 3' BAC end sequences were maintained in transgenic mice at a frequency of $31 / 60(52 \%)$. The ratio was significantly lower than that in the Ntng2 BAC deletion series (42/59, 71\%).

\section{Enhancer activities of Ntng 1 and Ntng2 ECRs}

Findings from the Ntng1 and Ntng2 BAC deletion series suggest that segment II in the Ntng2 and segment II and VI in the Ntng1 carry physiologically significant cis-regulatory elements. Based on comparisons among mouse, rat, human, chimp, rhesus, cow, dog, and chicken genomes, 


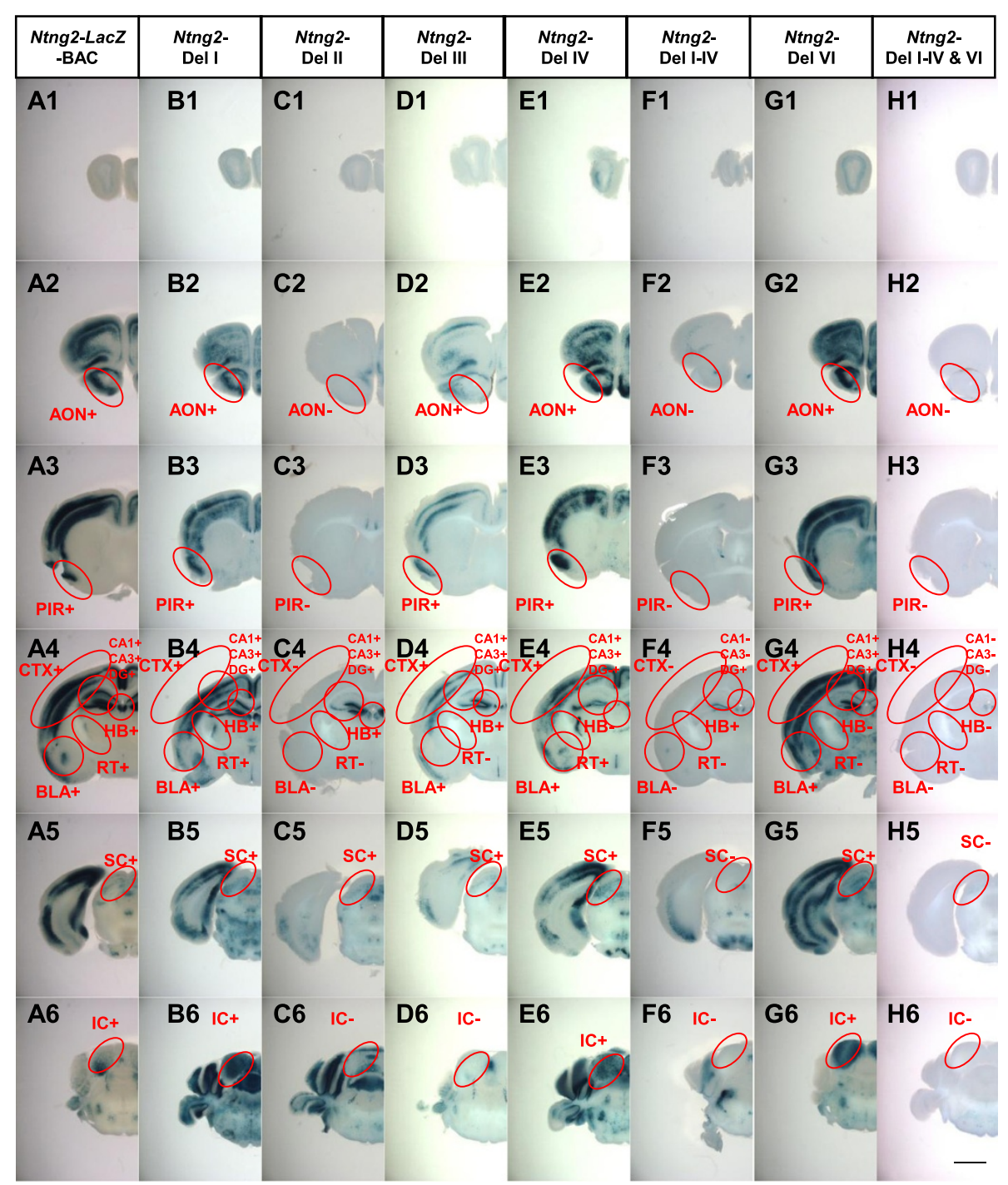

Figure 4 LacZ reporter expression profiles in the Ntng2-BAC deletion series. Brains from transgenic mice at P21 carrying Ntng2-BAC-LacZ (column A), Ntng2-Del I (B), II (C), III (D), IV (E), I-IV (F), VI (G), and I-IV\&VI (H) reporter constructs were sliced at $400 \mu \mathrm{m}$ and stained for LacZ activity. Images of the stained slices are arranged from anterior (Top) to posterior (Bottom). Red circles indicate the regions evaluated for LacZ activity. Abbreviations of the brain regions are the same as those in Figure 1. The $(+)$ and $(-)$ symbols indicate that the given construct induced expression of LacZ at a frequency over the criterion (50\% of transgenic founders) or not. Scale bar: $2.0 \mathrm{~mm}$ for all panels.

we focused on the most prominent ECRs; i.e., Ntng2ECR1, Ntng1-ECR1, and Ntng1-ECR2 (Additional files 4 and 6 Figures S2-S3). Ntng2-ECR1 in segment II locates at the $-68-\mathrm{kb}$ of TSS. Ntng1-ECR1 and ECR2 locate at the $-65-\mathrm{kb}$ of TSS in segment II and the $+11-\mathrm{kb}$ of TSS in segment VI, respectively. We evaluated the enhancer activities of these ECRs using a transgenic mouse enhancer assay in which the activity of each sequence was assessed through a $L a c Z$ reporter gene coupled to a minimal Hsp 68 promoter. Three constructs, Ntng2-ECR1-LacZ, Ntng1-ECR1-LacZ, and Ntng1-ECR2-LacZ were generated and injected into the pronuclei of fertilized eggs. $\mathrm{X}$-gal staining was performed to analyze the expression of $L a c Z$ in transgenic founders at P21. The results of these ECR-LacZ transgenic mice are shown in Figure 8 and Additional file 7: Table S4.

Transgenic mouse lines carrying the Ntng2-ECR1-LacZ construct had $L a c Z$ expression in the deeper cerebral layer and cerebellum (Figure 8A, Additional file 7: Table S4). The findings suggest that Ntng2-ECR1, located at the $-68 \mathrm{~kb}$ region in Ntng2 segment II, contains an enhancer responsible for the transcriptional regulation of Ntng2 in the cerebral cortex.

Transgenic lines carrying the Ntng1-ECR1-LacZ construct had LacZ expression in the regions of endogenous Ntng1 expression, such as the cerebral cortex layer $\mathrm{V}$, anterior 

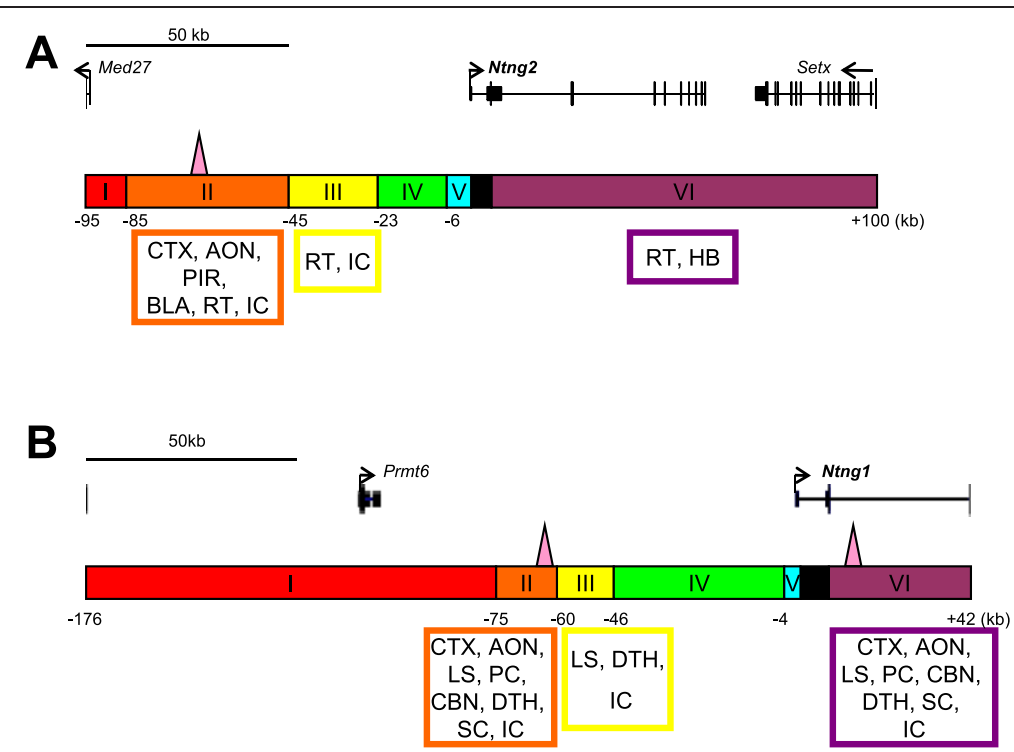

Figure 5 Summary of regulatory regions required for Ntng1 and Ntng2 expression. Genomic organization and genomic segments I-VI along with Ntng2 (A) and Ntng1 (B) gene organization are shown. Brain regions positively regulated by the genomic segments are indicated within the designated colored boxes at the bottom. The locations of Ntngl and Ntng2 enhancers identified are indicated by pink triangles. The combinations of cis-elements that locate distantly and widely from the TSS determine differential expression patterns of the vertebrate-specific paralogs, Ntng1 and Ntng2.

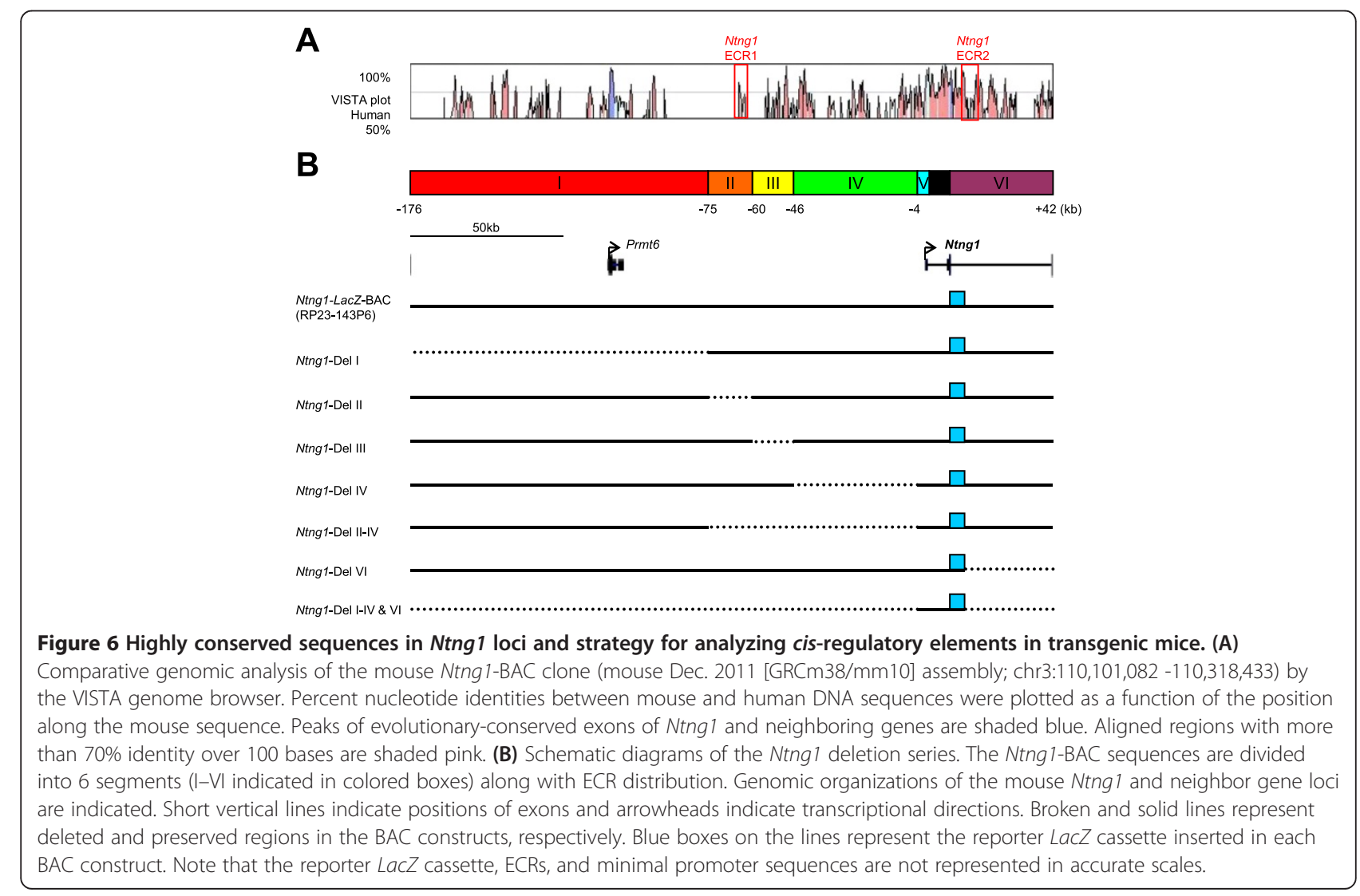




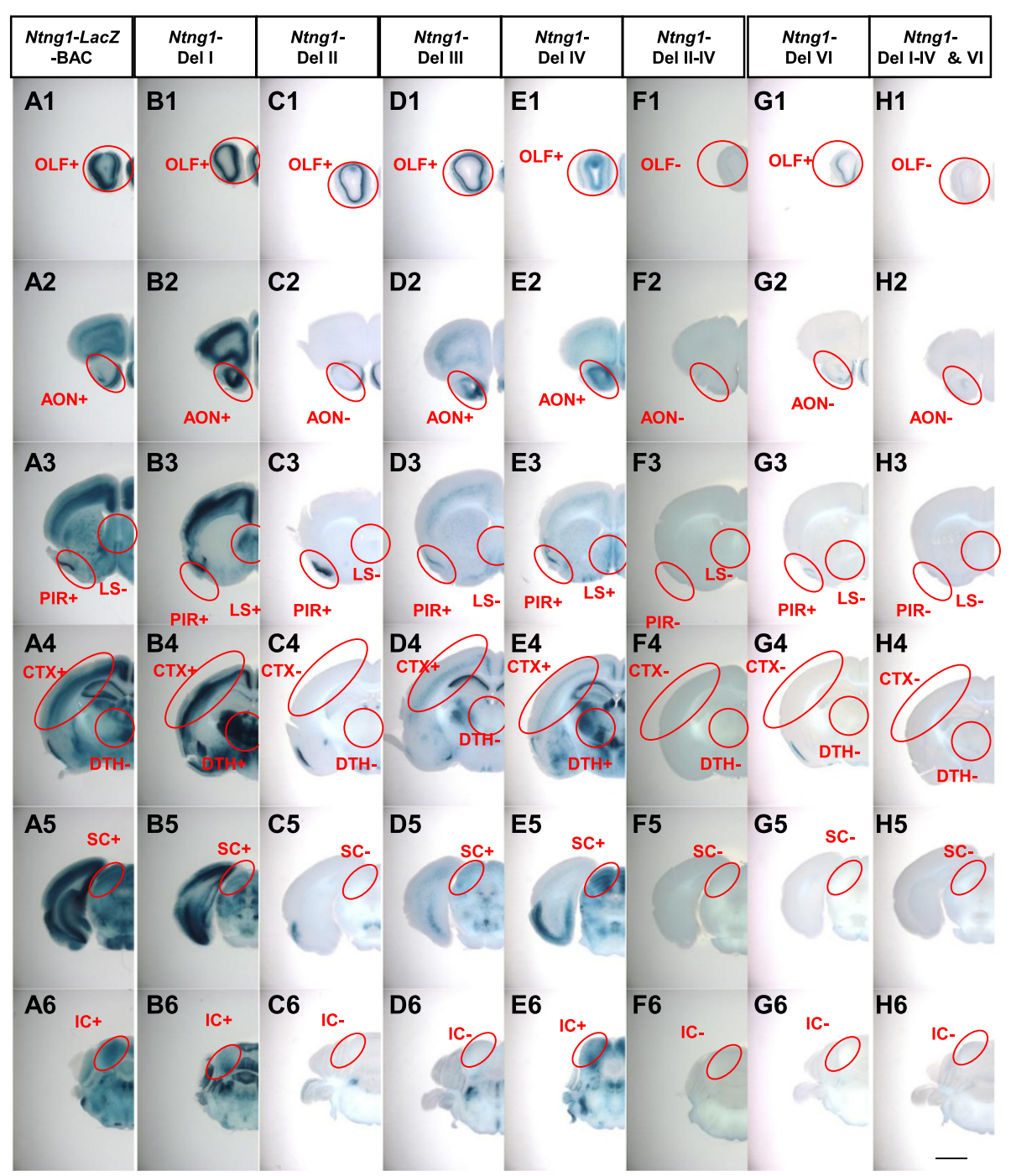

Figure 7 LacZ reporter expression profiles in the Ntng1-BAC deletion series. Brains from transgenic mice at P21 carrying Ntng1-BAC-LacZ (column A), Ntng1-Del I (B), II (C), III (D), IV (E), II-IV (F), VI (G), and I-IV\&VI (H) reporter constructs were sliced at 400 um and stained for LacZ activity. Images of the stained slices are arranged from anterior (Top) to posterior (Bottom). Red circles indicate the regions evaluated for $L a c Z$ activity. Abbreviations of the brain regions are the same as those in Figure 1. The $(+)$ and $(-)$ symbols indicate that the given construct-induced expression of LacZ at the frequency over the criterion (50\% of transgenic founders) or not. Scale bar: $2.0 \mathrm{~mm}$ for all panels.

olfactory nucleus, lateral septal nucleus, Purkinje cells, dorsal thalamus, superior colliculus, and inferior colliculus (Figure 8B, Additional file 7: Table S4). In transgenic lines carrying the Ntng1-ECR2-LacZ construct, however, no significant $L a c Z$ expression was detected in any brain region (Figure 8C, Additional file 7: Table S4). Therefore, these findings suggest that Ntng1-ECR1, located at the $-65-\mathrm{kb}$ region in Ntng1 segment II, but not Ntng1-ECR2, contains a physiologically significant enhancer responsible for the transcriptional regulation of endogenous Ntng1.

\section{Discussion}

In the present study, we investigated the mechanisms responsible for the complementary expression patterns of Ntng1 and Ntng2 in the central nervous system. Analyses of reporter gene expression patterns for both genes using Ntng1- and Ntng2-LacZ-KI mice confirmed the largely non-overlapping expression patterns of Ntng1 and Ntng2. Analysis of the expression patterns in KI alleles in the presence or absence of netrin-G1 and netrin-G2 functional proteins allowed us to exclude the possibility that a feedback (or feedforward) mechanism mediates the characteristic expression patterns of these genes. We next examined the cis-acting transcriptional regulatory mechanisms of Ntng1 and Ntng2 using BAC transgenic mice and transgenic enhancer assays. The results obtained from a series of BAC clones carrying systematic deletions revealed a complex regulatory architecture that depended 


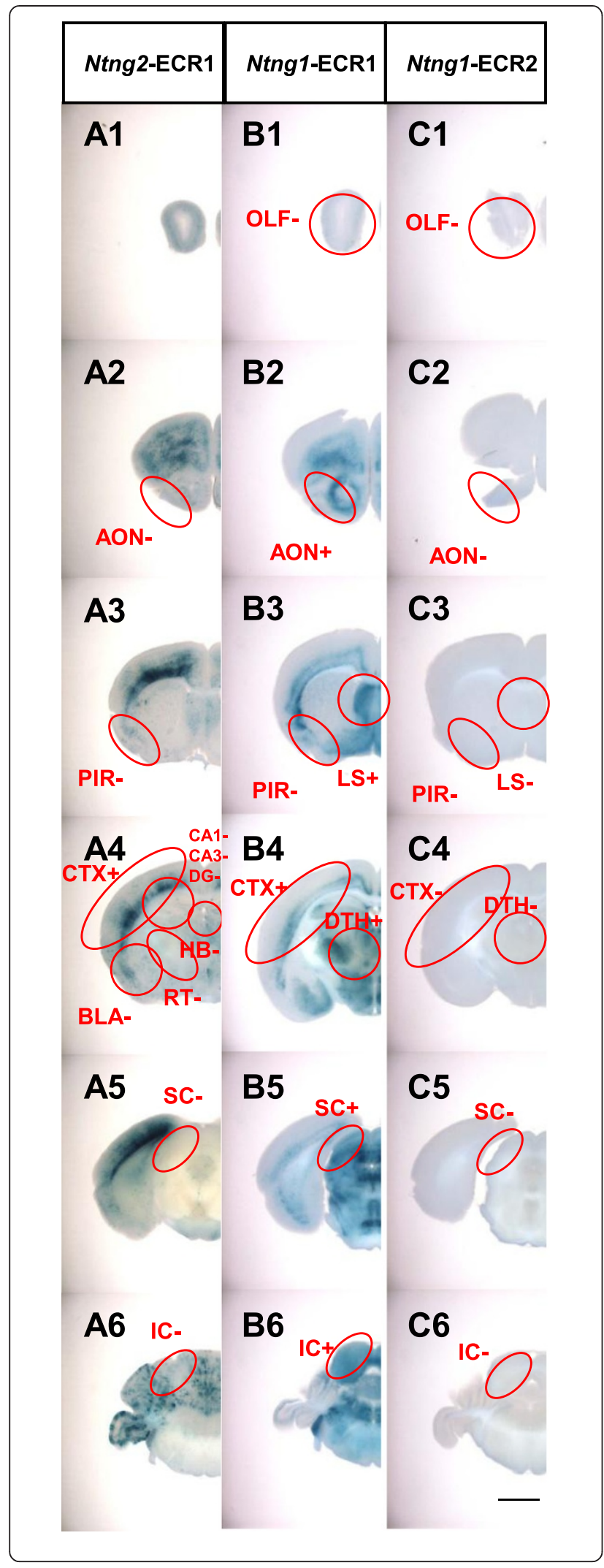

Figure 8 LaCZ reporter expression profiles in the Ntng 1 and

Ntng2 enhancer assays. Brains from transgenic mice at P21 carrying Ntng2-ECR1 (A column), Ntng1-ECR1 (B), and Ntng1-ECR2 (C) reporter constructs were sliced at $400 \mu \mathrm{m}$ and stained for $\mathrm{LaCZ}$ activity. Images of the stained slices are arranged from anterior (Top) to posterior (Bottom). Red circles guide the regions evaluated for $\mathrm{LaCZ}$ activity. Abbreviations of the brain regions are the same as those in Figure 1. The $(+)$ and $(-)$ symbols indicate that the given construct-induced expression of $\mathrm{LaCZ}$ at the frequency over the criterion (50\% of transgenic founders) or not. Scale bar: $2.0 \mathrm{~mm}$ for all panels.

on a diverse array of segments scattered over a 100-kb range of the locus in both genes. The Ntng2-segment II region contained major elements responsible for most brain regions. The Ntng1-segment II and VI regions cooperatively regulated Ntng1 expression in most brain regions. Furthermore, we successfully identified a $1.8-\mathrm{kb}$ enhancer of Ntng2 and a 2.0-kb enhancer of Ntng1 located at ECR regions $\sim 60 \mathrm{~kb}$ upstream from TSSs of both genes. These findings suggest that the complementary expression patterns of Ntng1 and Ntng2 are determined by transcriptional cis-elements widely scattered around the TSSs of these genes.

The high-resolution and highly sensitive analysis using LacZ-KI mice revealed a detailed picture of the complementary expression patterns of Ntng1 and Ntng2 in young adult mouse brains. Ntng1 is most abundantly expressed in brain regions involved in processing the early phases of sensory signal integration, such as the dorsal thalamus, superior colliculus, inferior colliculus, and olfactory bulb. The superior colliculus and lateral geniculate nuclei of the thalamus receive inputs from the retina and relay processed visual signals to the cerebral cortex. The inferior colliculus and medial geniculate nuclei of the thalamus process auditory signals, and the olfactory bulbs process olfactory signals. The dorsal thalamus is involved in processing all sensory signals, including secondary olfactory signals. Therefore, the regions that express Ntng1 are mainly involved in processing bottom-up signals. In contrast, Ntng2 is prominently expressed in the cortical layers, hippocampal circuits, habenula, claustrum, endopiriform nuclei, and reticular nuclei of the thalamus. These sites are involved in later stages of information processing, including top-down signals. The hippocampus plays a crucial role in several forms of learning and memory [34-38]. The frontal cortex is also involved in working memory [39-41]. Crick and Koch [42] proposed a role for the claustrum in integrated conscious perception as well as a role for the reticular nuclei of the thalamus in controlling attention by regulating sensory signal processing (i.e., the Searchlight hypothesis; [43]). The habenular complex has important roles in modulating learning, memory, and attention [44,45], and habenular dysfunction might be involved in schizophrenia and bipolar disorders [46]. Thus, the mechanisms that underlie the complementary 
expression patterns of Ntng1 and Ntng2 have had a large impact on the evolution of higher brain functions in vertebrates, particularly mammals.

Using the Ntng2-ECR1-LacZ construct, we induced reporter expression in the deep cortical layer. The BAC clone with a deletion of sequences containing the ECR1 failed to induce the expression of $L a c Z$ in the cortex. These findings suggest that the ECR1 at the 68-kb upstream region from the Ntng2 TSS works as a deep cortex layer-specific enhancer. The Ntng2-ECR1 sequences constituted a DNase I-hypersensitive site in adult mouse cortex (UCSC Genome Browser). DNase I-hypersensitive sites are regions of accessible chromatin, which are sensitive to cleavage by the DNase I enzyme and are markers of cis-regulatory elements [47]. Furthermore, we investigated transcription factors potentially involved in the 1.8-kb Ntng2-ECR1 sequences, using DiAlign TF [48] (Genomatix [http://www.genomatix.de/index.html]). DiAlign TF is a web-based tool for predicting transcription factor binding sites using cross-species comparisons. The Ntng2-ECR1 sequences were highly conserved among mouse, rat, human, chimpanzee, rhesus macaque, cow, dog, and chicken (Additional file 4: Figure S2), and contained 17 transcription factor binding sites within the sequences (Additional file 8: Figure S4). Among the sites, it is noteworthy the presence of CREB (cAMP response element-binding protein) site within the Ntng2-ECR1. CREB have crucial roles in activity-dependent transcriptional regulation in neurons [49]. We observed the activity-dependent increase in Ntng2 gene expression by age and behavioral experience in mice (unpublished observation by Pavel Prosselkov and SI). The CREB site in Ntng2-ECR1 may have a crucial role in the activitydependent Ntng2 expression and circuit plasticity. Taken together, these findings suggest that the Ntng2-ECR1 sequences act as an enhancer in adult mouse cortex.

In the case of Ntng1, we observed cooperative roles of Ntng1 segment II and VI for transcriptional regulation in most brain regions, suggesting that the Ntng1 regulatory mechanisms are highly complex. This observation may in part explain the reason of the longer genomic structure of Ntng1 (362 Kb, between the 1st and last exons) relative to that of Ntng2 (53 kb). Despite the complexity, however, we successfully identified enhancer activity in the Ntng1-ECR1 sequences, located at the -65 -kb region in Ntng1 segment II. The Ntng1-ECR1 sequences are relatively well conserved among mammals, but not in chicken (Additional file 6: Figure S3). DiAlign TF analysis based on comparisons between mouse and dog predicted sites for a limited number of transcription factors in the Ntng1-ECR1 (Additional file 9: Figure S5). These findings suggest that the cis-mechanism for Ntng1 has a radical evolutional history relative to that of Ntng2-ECR1.
In the Ntng1 BAC deletion study, Ntng1-Del II and Ntng1-Del VI transgenic mice showed a highly restricted expression pattern in the olfactory bulb (mitral cells) and piriform cortex. Thus, the expression of Ntng1 is separately regulated in the olfaction and other sensory modality pathways. The cis-mechanisms of Ntng1 make a physiologically significant contribution to elaborating the perception of multiple modalities in vertebrates/mammals. Practically, the Ntng1-Del II can be used as a useful transgenic vector in mice. Indeed, using the Ntng1-Del II, we successfully established an olfactory bulb and piriform cortex-specific Cre mouse line (Additional file 10: Figure S6).

Recent genome studies reported the whole genome sequences of the ascidian and amphioxus of chordates and the lamprey of early vertebrates [50-52]. The ancestral single Ntng ortholog (EntrezGene ID: 100178743) emerged in the ascidian genome in the evolutionary process. Ntng1 (Ensembl Gene ID: ENSPMAG00000002937) and Ntng2 (Ensembl Gene ID: ENSPMAG00000003799) are found in the lamprey genome, which underwent the first round of whole genome duplication after divergence from chordates. Therefore, it is likely that ancestral Ntng was divided into genes, Ntng1 and Ntng2, during the first round of whole genome duplication. It is noteworthy that Ntng1-ECR1 and Ntng2-ECR1 are equally distant from the TSSs. We argue that the cis-elements of Ntng1 and Ntng2 co-evolved in accordance with the duplication-degeneration-complementation model [53,54]. Differential behavioral phenotypes of Ntng1-KO and Ntng2-KO mice [13] clearly reveal the remarkable molecular evolution of Ntng1 and Ntng2.

\section{Conclusions}

Our findings suggest that the complementary expression patterns of Ntng1 and Ntng2 are determined by combinations of cis-regulatory elements that are widely scattered in these loci. The cis-regulatory elements in adult mouse brain characterized in this study will help facilitate the development of novel genetic tools for functionally dissecting neural circuits to better understand vertebrate brain function.

\section{Methods}

\section{Gene-targeted mice}

Ntng1-LacZ-KI and Ntng2-KO mice were described previously [12]. Using the same target sequences, we generated Ntng2-LacZ-KI mice and Ntng1-tauGFP-KI mice, which carried either a NLS-LacZ-polyA cassette or a tau and green fluorescent protein (GFP) fusion gene-polyA cassette within the exon 2 coding regions of Ntng1 or Ntng2. Mice with these mutations do not express endogenous netrin-G1 or netrin-G2. To genotype the KO and KI mice, DNA was extracted from the tails using a REDExtract-N-Amp Tissue PCR Kit (Sigma-Aldrich Chemical Co., St. Louis, MO, 
USA). The $L a c Z$ transgene was detected by polymerase chain reaction $(\mathrm{PCR})$ of the tail DNA with 30 cycles of $95^{\circ} \mathrm{C}$ for $1 \mathrm{~min}, 60^{\circ} \mathrm{C}$ for $1 \mathrm{~min}$, and $72^{\circ} \mathrm{C}$ for $1 \mathrm{~min}$. A pair of primers (Primer Nos. 1 and 2; Additional file 11: Table S5) was used to amplify the 515-bp LacZ region. The tau-GFP transgene was detected by PCR of the tail DNA under the same conditions. A pair of primers (Primer Nos. 3 and 4; Additional file 11: Table S5) was used to amplify the 303-bp tau-GFP region. The mice were maintained in the RIKEN animal facility. Mouse genotypes were determined by PCR using primers (Primer Nos. 1, 2, 5-9; Additional file 11: Table S5). Primer pairs of Ntng1 check up2/Ntng1 check down2 and LacZ1/LacZ2 yielded 199-bp and 515-bp fragments from the wild-type and Ntng1 KO allele, respectively. Ntng2 check up, Ntng2 check up2, and Ntng2 check down3 were used for genotyping the Ntng2 KO line. Ntng2 check up/Ntng2 check down3 and Ntng2 check up2/Ntng2 check down3 amplified 936-bp and 407-bp fragments from the wild-type and Ntng2 KO allele, respectively. All experimental protocols were approved by the RIKEN Institutional Animal Care and Use Committee.

\section{BAC clones}

Mouse BAC clones containing Ntng1 (RP23-143P6) and Ntng2 (RP23-417D10) from the C57BL/6 J mouse BAC library were purchased from the BACPAC Resource Center [http://bacpac.chori.org/home.htm]. In this study, BAC clones RP23-143P6, and RP23-417D10 were renamed Ntng1-BAC, and Ntng2-BAC, respectively. Ntng1-BAC covered a 218-kb region (mouse Dec. 2011 [GRCm38/mm10] assembly; chr3:110,101,082 -110,318,433) of the locus, representing the $176-\mathrm{kb}$ upstream $(-176 \mathrm{~kb})$ and $42-\mathrm{kb}$ downstream $(+42 \mathrm{~kb})$ sequences of Ntng1 TSS. Ntng2-BAC covered a 195-kb region (mouse Dec. 2011 [GRCm38/ mm10] assembly; chr2:29,153,280-29,347,847) of the locus, representing the sequences of Ntng2 TSS from $-95 \mathrm{~kb}$ to $+100 \mathrm{~kb}$.

\section{Modification of BAC clones}

BAC clones were modified using the Red/ET recombination technique (Gene Bridges, Dresden, Germany), which is based on homologous recombination aided by the inducible Red/ET recombination machinery. The NLSLacZ-pA-FRT5-Kan-FRT5 reporter cassette contains NLS-LacZ-pA and a prokaryotic promoter-driven kanamycin resistance gene (Kan) flanked by FRT5 sites $[55,56]$. To construct this cassette, the NLS-LacZ-pA cassette from the BINLacZ plasmid [57] was subcloned into an FRT5-flanked Kan vector (pCR-FRT5-Kan-FRT5) that was generated by inserting the PCR-amplified FRT5-flanked Kan cassette into a pCR-Blunt II-TOPO plasmid (Invitrogen, Carlsbad, CA, USA). The NLS-LacZ-pA-FRT5-Kan-FRT5 reporter cassette was amplified by PCR using Phusion High-Fidelity
DNA Polymerase (Finnzymes, Espoo, Finland). The primer sequences used (Primer Nos. 10-13) are shown in Additional file 11: Table S5. For in-frame replacement of the exon containing the Ntng1 or Ntng2 translation initiation codon (ATG) by the reporter cassette in the two BAC clones, each primer was designed to have 5' 60 nucleotide sequences corresponding to the BAC sequences and 3' 20 nucleotide sequences corresponding to the reporter cassette sequences. The PCR products were treated with $D p n$ I to selectively digest the template plasmids and then purified by ethanol precipitation. E. coli cells carrying BAC were transformed with a Red/ET expression plasmid, pSC101-BAD-gbaA (Gene Bridges), and recombination with the reporter cassette (NLS-LacZ-pAFRT5-Kan-FRT5) was subsequently induced. Successfully recombined colonies were identified by screening for kanamycin resistance, followed by PCR to ensure homologous recombination. The Kan cassette was subsequently removed from the recombined BAC clones by introducing the Flp recombinase expression plasmid, 706-FLP (Gene Bridges), into the bacterial cells. The resulting $\mathrm{BAC}$ transgene construct was verified by PCR, restriction fragment length polymorphisms, and DNA sequencing analyses.

A BAC deletion series was also generated using the Red/ET recombination system. The pCR-FRT-Amp-FRT plasmid was generated by inserting the PCR-amplified FRT-flanked Amp cassette into a pCR-Blunt II-TOPO plasmid (Invitrogen). FRT-flanked Amp-targeting fragments were amplified by PCR using Phusion High-Fidelity DNA Polymerase, 80-mer primers (Primer Nos. 17-33; Additional file 11: Table S5), and the pCR-FRT-Amp-FRT plasmid as a template. Subcloning from BAC constructs for Ntng1-Del I-IV \&VI and Ntng2-Del I-IV \&VI was also performed using the Red/ET recombination system. Fragments from $-4 \mathrm{~kb}$ (Ntng1) or $-6 \mathrm{~kb}$ (Ntng2) of TSSs to the 3' end of the NLS-LacZ-pA cassette from Ntng1-LacZBAC and Ntng2-LacZ-BAC constructs were subcloned into a pDEST R4-R3 vector (Invitrogen), containing the ampicillin resistance gene $(A m p)$ and pUC origin of replication. The pDEST R4-R3 linear vector fragment was amplified by PCR using Phusion High-Fidelity DNA Polymerase (Finnzymes) and primers (Primer Nos. 14-16; Additional file 11: Table S5).

The Ntng1-Del II-Cre BAC transgenic construct was also generated using the Red/ET system. The NLS-CrepA cassette was replaced the LacZ cassette of Ntng1-Del II construct.

Modified BAC clones were propagated in $2 \times 400 \mathrm{ml}$ of liquid culture and purified using a QIAGEN LargeConstruct Kit (QIAGEN, Hilden, Germany) according to the manufacturer's protocol. Correctly modified BACs were verified by conventional and pulsed-field gel analysis of restriction digests to confirm expected banding patterns 
and direct BAC sequencing at a vector-insert junction and $L a c Z$ cassette. All BAC reporter constructs were linearized by $A s c I$ digestion and purified for microinjection on sepharose columns as described previously [58].

\section{Generation of Ntng-ECR-Hsp-LacZ constructs}

Ntng-ECR-Hsp68-LacZ transgenes were generated as follows. PCR products corresponding to genomic sequences of Ntng1-ECR1 (2.0 kb), Ntng1-ECR2 (5.5 kb), and Ntng2ECR1 (1.8 kb) were amplified using Ntng1- or Ntng2-BAC DNA as the template, Phusion High-Fidelity DNA Polymerase, and primers (Primer Nos. 34-39; Additional file 11: Table S5). Each PCR product was ligated into the ASS-Hsp-LacZ-pA vector in the forward orientation to create ECR-Hsp-LacZ transgenes [59,60]. All ECR plasmids were verified by analysis of restriction enzyme digestion and DNA sequencing. Plasmid DNAs were purified using NucleoBond PC 500 (Macherey-Nagel) and digested with Not1. For injection, an ECR-Hsp-LacZ cassette without a vector fragment was isolated by agarose gel electrophoresis and purified using QIAEX II (QIAGEN). All ECR-Hsp-LacZ constructs were verified by analysis of restriction digests and direct sequencing.

\section{Generation of transgenic mice}

The purified DNA fragment $(1.0 \mathrm{ng} / \mu \mathrm{l})$ was injected into the pronuclei of fertilized eggs of C57BL/6 J $\times$ DBA/2 J F1 hybrid mice to generate transgenic mice. To genotype the transgenic mice, DNA was extracted from mouse tail samples collected at P10 using the REDExtract-N-Amp Tissue PCR Kit (Sigma Chemical Co.), and $L a c Z$ sequences were detected by PCR as described above. Cre transgenic mice were genotyped by PCR, using the primers (Primer Nos. 42 and 43; Additional file 11: Table S5), producing a 108-bp fragment from the Cre allele. For BAC transgenic organisms, the presence of pBACe3.6 BAC vector sequences immediately upstream or downstream of the Asc I site was further examined by PCR to minimize the possibility that large deletions occurred in the BAC integrated into the chromosome. LacZ expression analyses were performed for independent transgenic founders.

\section{X-gal staining}

For X-gal staining of the brain, mice were anesthetized by an intraperitoneal injection of 2.5\% 2,2,2-tribromoethanol at P21 and their brains were fixed with $40 \mathrm{ml}$ of $4 \%$ paraformaldehyde in phosphate-buffered saline (PBS) via transcardial perfusion at $4^{\circ} \mathrm{C}$ for $10 \mathrm{~min}$ after washing out the blood with physiologic saline. The fixed brains were sliced (thickness, $400 \mu \mathrm{m}$ ) using a Microslicer DTK-1000 (Dosaka EM, Kyoto, Japan) and placed in X-gal staining solution (0.1 M phosphate buffer [pH 7.5], $20 \mathrm{mM}$ Tris- $\mathrm{HCl}$ $[\mathrm{pH} 7.5], 5 \mathrm{mM} \mathrm{K}_{3} \mathrm{Fe}(\mathrm{CN})^{6}, 5 \mathrm{mM} \mathrm{K}_{4} \mathrm{Fe}(\mathrm{CN})^{6}, 2 \mathrm{mM}$ $\mathrm{MgCl}_{2}, 1 \mathrm{mg} / \mathrm{ml} \mathrm{X-gal)}$ for over $15 \mathrm{~h}$ at $37^{\circ} \mathrm{C}$.
For X-gal staining of thin sections, fixed brains were removed and postfixed in the same fixative for $2 \mathrm{~h}$. The brains were cryoprotected overnight in $15 \%$ sucrose in PBS and then overnight in $30 \%$ sucrose in PBS at $4^{\circ} \mathrm{C}$. They were then mounted in Tissue-Tek OCT compound (Sakura Finetek, Torrance, CA, USA), and stored at $-80^{\circ} \mathrm{C}$ until sectioned. Cryostat sections $(25 \mu \mathrm{m})$ were cut and thaw-mounted on MAS-coated slides (Matsunami Glass, Osaka, Japan). The slices were dried at room temperature and then stained overnight at $37^{\circ} \mathrm{C}$ in $\mathrm{X}$-gal staining solution. After a brief rinse in PBS, the tissue was counterstained with hematoxylin (Sakura Finetek, Japan) for $2 \mathrm{~min}$. After a brief rinse in PBS, the tissue was dehydrated in an ethanol series (70\%, 85\%, 95\%, and 100\% ethanol), dehydrated in xylene, and mounted with Eukitt (O. Kindler $\mathrm{GmbH} \& \mathrm{Co}$., Freiburg, Germany). Images of the entire brain sections were captured using a NanoZoomer RS slide scanner (Hamamatsu Photonics, Shizuoka, Japan) at 200-fold magnification.

\section{Comparative genome analysis}

Comparative genome analysis of the mouse Ntng1- and Ntng2-BAC sequences was performed using the VISTA browser [http://genome.lbl.gov/vista/index.shtml] [32]. Analysis of Ntng1 and Ntng2 loci covered the Ntng1-BAC (RP23-143P6) sequence (mouse Dec. 2011 [GRCm38/ mm10] assembly; chr3:110,101,082 -110,318,433) and Ntng2-BAC (RP23-417D10) sequence (mouse Dec. 2011 [GRCm38/mm10] assembly; chr2:29,153,280-29,347,847), respectively, as the reference sequences. The reference sequences included Ntng1 (NM_133488.1), Prmt6 (NR_024139.1), Ntng2 (NM_133501.1), Setx (NM_198033.2), and Med27 (NM_026896.4). Multiple alignments of Ntng1 and Ntng2 regions in mice versus those of Ntng1 and Ntng2 regions in other vertebrates were performed using the shuffle-LAGAN program [61]. Multiple alignments of the following assemblies were used for this analysis: mouse (Dec. 2011 [GRCm38/mm10] assembly), rat (Mar. 2012 [RGSC 5.0/rn5] assembly), human (Feb. 2009 [GRCh37/hg19] assembly), chimpanzee (Feb. 2011 [CSAC 2.1.4/panTro4] assembly), rhesus macaque (Oct. 2010 [BGI CR_1.0/rheMac3] assembly), cow (Oct. 2011 [Baylor Btau_4.6.1/bosTau7] assembly), dog (Sep. 2011 [Broad CanFam3.1/canFam3] assembly), chicken (Nov. 2011 [ICGSC Gallis_gallus-4.0/galGal4] assembly), and zebrafish (Jul. 2010 [Zv9/danRer7] assembly). We used the default VISTA parameter settings. The conservation rate between mice and the other vertebrates was plotted as percentages, and only the genomic regions with $50 \%-100 \%$ conservation are shown in the Additional file 4: Figures S2 and Additional file 6: Figure S3.

Potential transcription factor-binding sites within Ntng2ECR1 and Ntng1-ECR1 were predicted with the DiAlign TF [48] (Genomatix [http://www.genomatix.de/index.html]). 
Selected groups for solution parameters of DiAlign TF were as follows: MatInspector library Version 9.1; matrix group, general core promoter elements and vertebrates; matrix similarity, optimized for function.

\section{Additional files}

Additional file 1: Table S5. Primer sequences.

Additional file 2: Figure S1. LacZ-stained brain sections of $\mathrm{Ntng} 1$ and Ntng2 LacZ KI mice at high magnification. (A-E) X-gal staining of Ntng 1-LacZ KI mice. (F-J) X-gal staining of Ntng2-LacZ KI mice. (A-C, E-H, J) Coronal sections $(25 \mu \mathrm{m})$ at P21. (D, I) Horizontal sections $(25 \mu \mathrm{m})$ at P21. Sections were lightly counterstained with hematoxylin. In the anterior olfactory nucleus, Ntng 1 was expressed in the neurons of dorsal-, lateral-, medial-part, and Ntng2 was expressed in the neurons of the external part. In the piriform cortex, Ntng 1 was expressed in the semilunar neurons of layer II, whereas Ntng2 was expressed in the pyramidal and polymorphic neurons of layer III. In the cerebral cortex, Ntngl was expressed in layer V neurons, whereas Ntng2 was expressed in neurons in layers II/III, IV, and VI. In the entorhinal area, Ntng 1 was expressed in layer II of the lateral entorhinal area (LEC) and layer III throughout the entorhinal area, and Ntng 2 was expressed in layer II of the medial entorhinal area (MEC) and layer III of the LEC. In the amygdala, Ntng1 was expressed in the lateral amygdala nucleus (LA), whereas Ntng2 was expressed in the basolateral amygdala nucleus (BLA). Scale bar: $1.0 \mathrm{~mm}$ for all panels.

Additional file 3: Table S2. Summary of LaCZ expression domains observed in the Ntng2 deletion series. The ratio of transgenic mice with reproducible reporter expression per all independent founders in regions expressing endogenous Ntng2 is shown. Blue cells: reproducible expression in more than half of all independent transgenic founders in the given brain region.

Additional file 4: Figure S2. Evolutionarily conserved region (ECR) in the mouse Ntng2 locus. Analysis of the Ntng2 locus covered by the Ntng2-BAC (mouse Dec. 2011 [GRCm38/mm10] assembly; chr2: 29,153,280-29,347,847). Comparative genomic analysis of the mouse Ntng2-BAC sequence by the VISTA genome browser (http://genome.lbl. gov/vista/index.shtml). Percent nucleotide identities between mouse and other species (rat, human, chimp, rhesus, cow, dog, chicken, and zebrafish; from top to bottom) are plotted as a function of the position along the mouse sequence. Peaks of evolutionarily conserved overlapping exons of Ntng 2 and neighboring genes are shaded blue. Aligned regions with more than $70 \%$ identity over 100 bases are shaded pink. Ntng2 ECR1, indicated by the red rectangle, represents the most highly conserved region and locates within segment II (Figures 3 and 5).

Additional file 5: Table S3. Summary of LacZ expression domains observed in the Ntngl deletion series. The ratio of transgenic mice with reproducible reporter expression per all independent founders in regions of endogenous Ntng1 expression is shown. Blue cells: reproducible expression in more than half of all independent transgenic founders in the given brain region. Light blue cells: although the ratio of reproducible expression did not fulfill the criteria, the highly restricted expression pattern might be significant.

Additional file 6: Figure S4. Multiple sequence alignment and candidate transcription-factor binding sites in Ntng2-ECR1. Sequence comparisons of the Ntng2 enhancer Ntng2-ECR1 sites between mouse, rat, human, chimp, rhesus, cow, dog, and chicken species. Putative transcription factor binding sites are highlighted in the differential colors, respectively. Seventeen potential highly conserved binding sites were identified (searched results are from DiAlign TF).

Additional file 7: Table S4. Summary of LacZ expression domains observed in the Ntng 2 and Ntng 1 enhancer analysis series. The ratio of transgenic mice with reproducible reporter expression in regions expressing endogenous Ntng2 (left) and Ntng 1(right) is shown. Blue cells: the results were consistent with those from the deletion series.

Additional file 8: Figure S3. ECR in the mouse Ntng 1 locus. Analysis of the Ntng1 locus covered by the Ntng1-BAC (mouse Dec. 2011
[GRCm38/mm10] assembly; chr3:110,101,082 -110,318,433) by the VISTA genome browser (http://genome.lbl.gov/vista/index.shtml). Percent nucleotide identities between mouse and other species (rat, human, chimp, rhesus, cow, dog, chicken, and zebrafish; from top to bottom) are plotted as a function of the position along the mouse sequence. Peaks of evolutionary conservation overlapping exons of Ntng 1 and neighboring genes are shaded blue. Aligned regions with more than $70 \%$ identity over 100 bases are shaded pink. Ntng 1 ECR1 and ECR2, indicated by the red rectangles, represent one of the most highly conserved regions and locate within segments II and VI, respectively (Figures 5 and 6).

Additional file 9: Figure S5. Multiple sequence alignment and candidate transcription-factor binding sites in Ntng 1-ECR1. Sequence comparisons of the Ntngl enhancer Ntng 1-ECR1 sites between mouse, rat, human, chimp, rhesus, cow, and dog species. Putative transcription factor binding sites are highlighted in the differential colors, respectively Eight potential highly conserved binding sites were identified (searched results are from DiAlign TF).

Additional file 10: Figure S6. Recombination patterns in Ntng 1-Del II-Cre: Rosa-NLSLacZ mice. (A-H) LacZ activies (blue signals) of Ntng1-Del II-Cre :Rosa-NLSLacZ [44] mice. Coronal sections (A-F: $400 \mu \mathrm{m}, \mathrm{G}-\mathrm{H}: 25 \mu \mathrm{m}$ ) were stained with $\mathrm{X}$-gal solution. Sections were lightly counterstained with hematoxylin. Red circles indicated the region-specific Lac $Z$ activities. In the olfactory areas, Cre was highly expressed in the olfactory bulb mitral cells. In the piriform cortex, Cre was expressed in the semilunar neurons of layer II. Scale bar: $2.0 \mathrm{~mm}$ for A-F panels, $500 \mu \mathrm{m}$ for G-H panels.

Additional file 11: Table S1. Summary of LacZ expression in Ntng 1 and Ntng2 LacZ KI mice. The in situ hybridization data of Ntng1 and Ntng2 genes in P28 mice were quoted from Allen Brain Atlas [62] [http://www. brain-map.org/]. (-), no expression; (+), weak expression; (++), moderate expression; $(+++)$, strong expression; (n.d.), no data.

\section{Abbreviations}

BAC: Bacterial artificial chromosome; kb: Kilo base; Ntng1: Netrin-G1; Ntng2: Netrin-G2; KO: Knock-out; ECR: Evolutionarily conserved region; LacZ: E.coli beta-galactosidase; Kl: Knock-in; TSS: Transcription start site; NLS: Nuclear localization signal; pA: Polyadenylation signal; X-gal: 5-bromo-4chloro-3-indolyl- $\beta$-d-galactoside; P21: Postnatal day 21; OR: Olfactory receptor; GFP: Green fluorescent protein; PCR: Polymerase chain reaction; Kan: Kanamycin resistance gene; ATG: Translation initiation codon; Amp: Ampicillin resistance gene; PBS: Phosphate-buffered saline; BR: Brain regions; $A D$ : Anterodorsal nucleus of the thalamus; $A O N$ : Anterior olfactory nucleus; BC: Bergmann glia cells; BLA: Basolateral amygdala nucleus; CA1: Cornu ammonis 1 field of the hippocampus; CA3: Cornu ammonis 3 field of the hippocampus; CBN: Cerebellar nuclei; CLA: Claustrum; CN: Cochlear nuclei; CTX: Cerebral cortex; CU: Cuneate nucleus; DG: Dentate gyrus; DMX: Dorsal motor nucleus of the vagus nerve; DTH: Dorsal thalamus; ENT: Entorhinal area; Epd: Endopiriform nucleus, dorsal part; HB: Habenula; IC: Inferior colliculus; isl: Olfactory tubercle, islands of Calleja; IO: Inferior olivary complex; LA: Lateral amygdala nucleus; LRN: Lateral reticular nucleus; LS: Lateral septal nucleus; OLFgl: Olfactory bulb, glomerular layer; OLFmi: Olfactory bulb, mitral layer; PAG: Periaqueductal gray, dorsal division; PC: purkinje cell; PIR: Piriform area; PG: Pontine gray; PRT: Pretectal region; PSV: Principal sensory nucleus of the trigeminal; RAmb: Midbrain raphe nuclei; RN: Red nucleus; RT: Reticular nucleus of the thalamus; SC: Superior colliculus; SPV: Spinal nucleus of the trigeminal; STN: Subthalamic nucleus; SUB: Subiculum; SUM: Supramammillary nucleus; TRN: Tegmental reticular nucleus; TT: Taenia tecta; III: Oculomotor nucleus; V: Trigeminal motor nucleus; VII: Facial motor nucleus; XII: Hypoglossal nucleus.

\section{Competing interests}

The authors declare that they have no competing interests.

\section{Authors' contributions}

KY performed the experiments. KY and SI designed the experiments and analyzed data. SN provided the Ntng 1 and Ntng 2 gene-targeted mice. KY and SK generated and analysed a Cre transgenic mouse line. TO analyzed data. KY and SI wrote the manuscript. All authors have read and approved the manuscript. 


\section{Acknowledgements}

We thank the staff of the Research Resources Center of the RIKEN Brain Science Institute for animal care and technical support, Hiroshi Sasaki for the gift of the Ass-Hsp-LacZ plasmid, Jun Aruga and Takashi Kondo for helpful discussions, and all members of the Laboratory for Behavioral Genetics for stimulating discussion and technical support. This work was partly supported by a Grant-in-Aid for Scientific Research (A) from the Ministry of Education, Culture, Sports, Science and "Funding Program for World-Leading Innovative R\&D on Science and Technology (FIRST Program)" initiated by the Counci for Science and Technology Policy (CSTP).

\section{Author details}

'RIKEN Brain Science Institute, 2-1 Hirosawa, Wako, Saitama, Japan. ${ }^{2}$ Department of Molecular Immunology, University of Tokyo, 1-1-1 Yayoi, Bunkyo, Tokyo, Japan.

Received: 24 December 2013 Accepted: 9 March 2014 Published: 19 March 2014

\section{References}

1. Lewis DA, Sweet RA: Schizophrenia from a neural circuitry perspective: advancing toward rational pharmacological therapies. J Clin Invest 2009, 119:706-716.

2. Walsh CA, Morrow EM, Rubenstein JL: Autism and brain development. Cell 2008, 135:396-400.

3. Lettice LA, Heaney SJ, Purdie LA, Li L, de Beer P, Oostra BA, Goode D, Elgar $G$, Hill RE, de Graaff E: A long-range Shh enhancer regulates expression in the developing limb and fin and is associated with preaxial polydactyly. Hum Mol Genet 2003, 12:1725-1735.

4. Nakashiba T, Ikeda T, Nishimura S, Tashiro K, Honjo T, Culotti JG, Itohara S: Netrin-G1: a novel glycosyl phosphatidylinositol-linked mammalian netrin that is functionally divergent from classical netrins. J Neurosci 2000, 20:6540-6550.

5. Nakashiba T, Nishimura S, Ikeda T, Itohara S: Complementary expression and neurite outgrowth activity of netrin-G subfamily members. Mech Dev 2002, 111:47-60

6. Yin $Y$, Miner JH, Sanes JR: Laminets: laminin- and netrin-related genes expressed in distinct neuronal subsets. Mol Cell Neurosci 2002, 19:344-358.

7. Ishii N, Wadsworth WG, Stern BD, Culotti JG, Hedgecock EM: UNC-6, a laminin-related protein, guides cell and pioneer axon migrations in $\mathrm{C}$. elegans. Neuron 1992, 9:873-881.

8. Livesey FJ: Netrins and netrin receptors. Cell Mol Life Sci 1999, 56:62-68.

9. Serafini T, Kennedy TE, Galko MJ, Mirzayan C, Jessell TM, Tessier-Lavigne M: The netrins define a family of axon outgrowth-promoting proteins homologous to C. elegans UNC-6. Cell 1994, 78:409-424.

10. Lin JC, Ho WH, Gurney A, Rosenthal A: The netrin-G1 ligand NGL-1 promotes the outgrowth of thalamocortical axons. Nat Neurosci 2003, 6:1270-1276.

11. Kim S, Burette A, Chung HS, Kwon SK, Woo J, Lee HW, Kim K, Kim H, Weinberg RJ, Kim E: NGL family PSD-95-interacting adhesion molecules regulate excitatory synapse formation. Nat Neurosci 2006, 9:1294-1301.

12. Nishimura-Akiyoshi S, Niimi K, Nakashiba T, Itohara S: Axonal netrin-Gs transneuronally determine lamina-specific subdendritic segments. Proc Natl Acad Sci USA 2007, 104:14801-14806.

13. Matsukawa T, Goto H, Lujan R, Akiyoshi-Nishimura S, Zhang Q, Yaguchi K, Hashikawa T, Shigemoto R, Itohara S: Presynaptic netrin-G subtypes differentially control synaptic plasticity and behavior in mice. In Neuroscience. New Orleans, Louisiana: Society for Neuroscience; 2012.

14. Miyashita T, Nishimura-Akiyoshi S, Itohara S, Rockland KS: Strong expression of NETRIN-G2 in the monkey claustrum. Neuroscience 2005, 136:487-496.

15. Meerabux JM, Ohba H, Fukasawa M, Suto Y, Aoki-Suzuki M, Nakashiba T, Nishimura S, Itohara S, Yoshikawa T: Human netrin-G1 isoforms show evidence of differential expression. Genomics 2005, 86:112-116.

16. Aoki-Suzuki M, Yamada K, Meerabux J, Iwayama-Shigeno Y, Ohba H, Iwamoto K, Takao H, Toyota T, Suto Y, Nakatani N, Dean B, Nishimura S, Seki K, Kato T, Itohara S, Nishikawa T, Yoshikawa T: A family-based association study and gene expression analyses of netrin-G1 and -G2 genes in schizophrenia. Biol Psychiatry 2005, 57:382-393.

17. Fukasawa M, Aoki M, Yamada K, Iwayama-Shigeno Y, Takao H, Meerabux J, Toyota T, Nishikawa T, Yoshikawa T: Case-control association study of human netrin G1 gene in Japanese schizophrenia. J Med Dent Sci 2004, 51:121-128.

18. Ohtsuki T, Horiuchi Y, Koga M, Ishiguro H, Inada T, Iwata N, Ozaki N, Ujike H, Watanabe Y, Someya T, Arinami T: Association of polymorphisms in the haplotype block spanning the alternatively spliced exons of the NTNG1 gene at 1 p13.3 with schizophrenia in Japanese populations. Neurosci Lett 2008, 435:194-197.

19. Bisgaard AM, Rasmussen LN, Moller HU, Kirchhoff M, Bryndorf T: Interstitial deletion of the short arm of chromosome 1 (1p13.1p21.1) in a girl with mental retardation, short stature and colobomata. Clin Dysmorphol 2007, 16:109-112.

20. Borg I, Freude K, Kubart S, Hoffmann K, Menzel C, Laccone F, Firth H, Ferguson-Smith MA, Tommerup N, Ropers HH, Sargan D, Kalscheuer VM: Disruption of Netrin G1 by a balanced chromosome translocation in a girl with Rett syndrome. Eur J Hum Genet 2005, 13:921-927.

21. Eastwood SL, Harrison PJ: Decreased mRNA expression of netrin-G1 and netrin-G2 in the temporal lobe in schizophrenia and bipolar disorder. Neuropsychopharmacology 2008, 33:933-945.

22. Eastwood SL, Harrison PJ: Markers of glutamate synaptic transmission and plasticity are increased in the anterior cingulate cortex in bipolar disorder. Biol Psychiatry 2010, 67:1010-1016.

23. Gong S, Zheng C, Doughty ML, Losos K, Didkovsky N, Schambra UB, Nowak $\mathrm{NJ}$, Joyner A, Leblanc G, Hatten ME, Heintz N: A gene expression atlas of the central nervous system based on bacterial artificial chromosomes. Nature 2003, 425:917-925.

24. Tomishima MJ, Hadjantonakis AK, Gong S, Studer L: Production of green fluorescent protein transgenic embryonic stem cells using the GENSAT bacterial artificial chromosome library. Stem Cells 2007, 25:39-45.

25. Copeland NG, Jenkins NA, Court DL: Recombineering: a powerful new tool for mouse functional genomics. Nat Rev Genet 2001, 2:769-779.

26. Bejerano G, Siepel AC, Kent WJ, Haussler D: Computational screening of conserved genomic DNA in search of functional noncoding elements. Nat Methods 2005, 2:535-545.

27. Lenhard B, Sandelin A, Mendoza L, Engstrom P, Jareborg N, Wasserman WW: Identification of conserved regulatory elements by comparative genome analysis. J Biol 2003, 2:13.

28. Ureta-Vidal A, Ettwiller L, Birney E: Comparative genomics: genome-wide analysis in metazoan eukaryotes. Nat Rev Genet 2003, 4:251-262.

29. Serizawa S, Miyamichi K, Sakano H: One neuron-one receptor rule in the mouse olfactory system. Trends Genet 2004, 20:648-653.

30. Bergman $Y$, Cedar H: A stepwise epigenetic process controls immunoglobulin allelic exclusion. Nat Rev Immunol 2004, 4:753-761.

31. Mostoslavsky R, Alt FW, Rajewsky K: The lingering enigma of the allelic exclusion mechanism. Cell 2004, 118:539-544.

32. Zhang Y, Buchholz F, Muyrers JP, Stewart AF: A new logic for DNA engineering using recombination in Escherichia coli. Nat Genet 1998, 20:123-128.

33. Frazer KA, Pachter L, Poliakov A, Rubin EM, Dubchak I: VISTA: computational tools for comparative genomics. Nucleic Acids Res 2004, 32:W273-279.

34. Bliss TV, Collingridge GL: A synaptic model of memory: long-term potentiation in the hippocampus. Nature 1993, 361:31-39.

35. Jarrard LE: On the role of the hippocampus in learning and memory in the rat. Behav Neural Biol 1993, 60:9-26.

36. Nakashiba T, Young JZ, McHugh TJ, Buhl DL, Tonegawa S: Transgenic inhibition of synaptic transmission reveals role of CA3 output in hippocampal learning. Science 2008, 319:1260-1264.

37. Scoville WB, Milner B: Loss of recent memory after bilateral hippocampal lesions. J Neurol Neurosurg Psychiatry 1957, 20:11-21.

38. Squire LR, Stark CE, Clark RE: The medial temporal lobe. Annu Rev NeurosCi 2004, 27:279-306.

39. Courtney SM, Petit L, Maisog JM, Ungerleider LG, Haxby JV: An area specialized for spatial working memory in human frontal cortex. Science 1998, 279:1347-1351.

40. Goldman-Rakic PS: Circuitry of Primate Prefrontal Cortex and Regulation of Behavior by Representational Memory. In Handbook of Physiology, The Nervous System, Higher Functions of the Brain. Bethesda: American Physiological Society; 1987:37-417.

41. Passingham D, Sakai $K$ : The prefrontal cortex and working memory: physiology and brain imaging. Curr Opin Neurobiol 2004, 14:163-168

42. Crick FC, Koch $C$ : What is the function of the claustrum? Philos Trans $R$ SoC Lond B Biol Sci 2005, 360:1271-1279. 
43. Crick F: Function of the thalamic reticular complex: the searchlight hypothesis. Proc Natl Acad Sci USA 1984, 81:4586-4590.

44. Kobayashi Y, Sano Y, Vannoni E, Goto H, Suzuki H, Oba A, Kawasaki H, Kanba S, Lipp HP, Murphy NP, Wolfer DP, Itohara S: Genetic dissection of medial habenula-interpeduncular nucleus pathway function in mice. Front Behav Neurosci 2013, 7:17.

45. Lecourtier L, Kelly PH: A conductor hidden in the orchestra? Role of the habenular complex in monoamine transmission and cognition. Neurosci Biobehav Rev 2007, 31:658-672.

46. Caputo A, Ghiringhelli L, Dieci M, Giobbio GM, Tenconi F, Ferrari L, Gimosti E, Prato K, Vita A: Epithalamus calcifications in schizophrenia. Eur Arch Psychiatry Clin Neurosci 1998, 248:272-276.

47. Mercer TR, Edwards SL, Clark MB, Neph SJ, Wang H, Stergachis AB, John S, Sandstrom R, Li G, Sandhu KS, Ruan Y, Nielsen LK, Mattick JS, Stamatoyannopoulos JA: DNase I-hypersensitive exons colocalize with promoters and distal regulatory elements. Nat Genet 2013, 45:852-859.

48. Cartharius K, Frech K, Grote K, Klocke B, Haltmeier M, Klingenhoff A, Frisch M, Bayerlein M, Werner T: MatInspector and beyond: promoter analysis based on transcription factor binding sites. Bioinformatics 2005, 21:2933-2942.

49. Barco A, Marie H: Genetic approaches to investigate the role of CREB in neuronal plasticity and memory. Mol Neurobiol 2011, 44:330-349.

50. Dehal P, Satou Y, Campbell RK, Chapman J, Degnan B, De Tomaso A, Davidson B, Di Gregorio A, Gelpke M, Goodstein DM, Harafuji N, Hastings KE, Ho I, Hotta K, Huang W, Kawashima T, Lemaire P, Martinez D, Meinertzhagen IA, Necula S, Nonaka M, Putnam N, Rash S, Saiga H, Satake M, Terry A, Yamada L, Wang HG, Awazu S, Azumi K, et al: The draft genome of Ciona intestinalis: insights into chordate and vertebrate origins. Science 2002, 298:2157-2167.

51. Putnam NH, Butts T, Ferrier DE, Furlong RF, Hellsten U, Kawashima T, Robinson-Rechavi M, Shoguchi E, Terry A, Yu JK, Benito-Gutierrez EL, Dubchak I, Garcia-Fernandez J, Gibson-Brown JJ, Grigoriev IV, Horton AC, de Jong PJ, Jurka J, Kapitonov W, Kohara Y, Kuroki Y, Lindquist E, Lucas S, Osoegawa K, Pennacchio LA, Salamov AA, Satou Y, Sauka-Spengler T, Schmutz J, Shin IT, et al: The amphioxus genome and the evolution of the chordate karyotype. Nature 2008, 453:1064-1071.

52. Smith JJ, Kuraku S, Holt C, Sauka-Spengler T, Jiang N, Campbell MS, Yandell MD, Manousaki T, Meyer A, Bloom OE, Morgan JR, Buxbaum JD, Sachidanandam R, Sims C, Garruss AS, Cook M, Krumlauf R, Wiedemann LM, Sower SA, Decatur WA, Hall JA, Amemiya CT, Saha NR, Buckley KM, Rast JP, Das S, Hirano M, McCurley N, Guo P, Rohner N, et al: Sequencing of the sea lamprey (Petromyzon marinus) genome provides insights into vertebrate evolution. Nat Genet 2013, 45:415-421. 421e411-412.

53. Force A, Lynch M, Pickett FB, Amores A, Yan YL, Postlethwait J: Preservation of duplicate genes by complementary, degenerative mutations. Genetics 1999, 151:1531-1545.

54. Innan H, Kondrashov F: The evolution of gene duplications: classifying and distinguishing between models. Nat Rev Genet 2010, 11:97-108.

55. Schlake T, Bode J: Use of mutated FLP recognition target (FRT) sites for the exchange of expression cassettes at defined chromosomal loci. Biochemistry 1994, 33:12746-12751.

56. Seibler J, Schubeler D, Fiering S, Groudine M, Bode J: DNA cassette exchange in ES cells mediated by Flp recombinase: an efficient strategy for repeated modification of tagged loci by marker-free constructs. Biochemistry 1998, 37:6229-6234.

57. Sassa T, Gomi H, Itohara S: Postnatal expression of Cdkl2 in mouse brain revealed by LacZ inserted into the Cdkl2 locus. Cell Tissue Res 2004, 315:147-156.

58. Hammes A, Schedl A: Generation of transgenic mice from plasmids, BACs and YACs. In Mouse genetics and transgenics. Edited by Jackson IJ. aCMA. Leeds: Oxford University Press; 2000:217-245.

59. Rossant J, Zirngibl R, Cado D, Shago M, Giguere V: Expression of a retinoic acid response element-hsplacZ transgene defines specific domains of transcriptional activity during mouse embryogenesis. Genes Dev 1991, 5:1333-1344.

60. Sasaki H, Hogan BL: Enhancer analysis of the mouse HNF-3 beta gene: regulatory elements for node/notochord and floor plate are independent and consist of multiple sub-elements. Genes Cells 1996, 1:59-72.
61. Brudno M, Do CB, Cooper GM, Kim MF, Davydov E, Green ED, Sidow A, Batzoglou S: LAGAN and Multi-LAGAN: efficient tools for large-scale multiple alignment of genomic DNA. Genome Res 2003, 13:721-731.

62. Lein ES, Hawrylycz MJ, Ao N, Ayres M, Bensinger A, Bernard A, Boe AF, Boguski MS, Brockway KS, Byrnes EJ, Chen L, Chen TM, Chin MC, Chong J, Crook BE, Czaplinska A, Dang CN, Datta S, Dee NR, Desaki AL, Desta T, Diep E, Dolbeare TA, Donelan MJ, Dong HW, Dougherty JG, Duncan BJ, Ebbert AJ, Eichele G, Estin LK, et al: Genome-wide atlas of gene expression in the adult mouse brain. Nature 2007, 445:168-176.

doi:10.1186/1756-6606-7-19

Cite this article as: Yaguchi et al:: Identification of transcriptional regulatory elements for Ntng1 and Ntng2 genes in mice. Molecular Brain 2014 7:19.

\section{Submit your next manuscript to BioMed Central and take full advantage of:}

- Convenient online submission

- Thorough peer review

- No space constraints or color figure charges

- Immediate publication on acceptance

- Inclusion in PubMed, CAS, Scopus and Google Scholar

- Research which is freely available for redistribution 